\title{
THE DETERMINATION OF THE MAGNETIC INDUCTION IN STRAIGHT BARS.
}

\author{
By Charles W. Burrows.
}

\section{CONTENTS.}

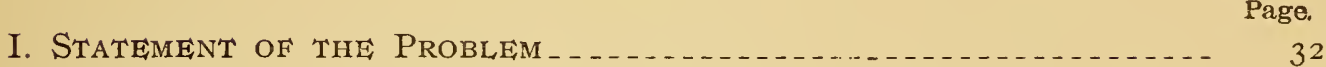

II. ThEORY OF a Distributed Magnetomotive ForCE........... 33

III. PRELIMINARY INVESTIGATIONS_..

I. Yoke Reluctance......... 35

2. Flux Distribution in the Iron . . . . 39

3. Leakage Flux in Air

4. Reactive Force of the Yokes _........ 45

5. Curved Yokes

6. Double Compensation ................ 50

7. Various Forms of Yokes ............ 52

8. Uniformity of Specimen _.

9. Distribution of Test Coils _...

IV. Direct Reading Method of Measuring the Magnetomotive Force - 63

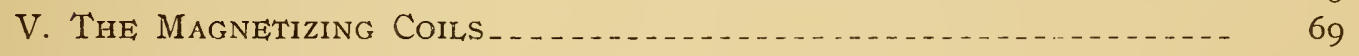

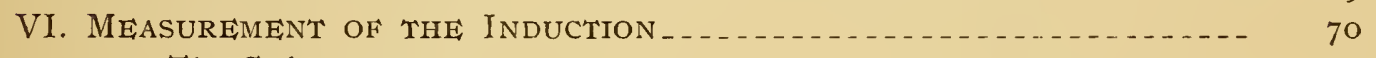

I. The Galvanometer $\ldots$

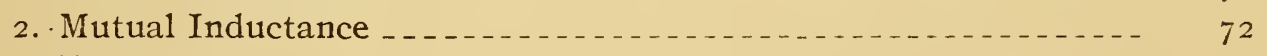

3. The Current ..... 73

4. Test Coils _..

5. Cross Section of Specimen _.

6. Air Flux . .

7. Zero Method

8. Variable Mutual Inductance................. 78

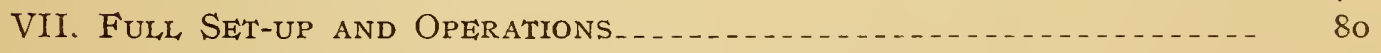

I. For Rings and Uncompensated Bars and Yoke_......... 84

2. Compensated Bar and Yoke ........................ 85

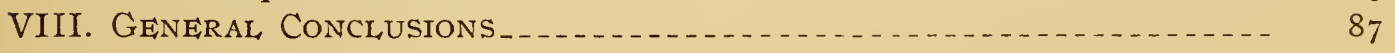

The present paper is a description of a method of making precision magnetic measurements in use at the Bureau of Standards, and also of some of the investigations which were made during the development of the method. 


\section{STATEMENT OF THE PROBLEM.}

The problem of the measurement of the magnetic induction in a given specimen consists in the simultaneous determination of the magnetic flux density and the magnetizing force at a given point. The difficulties depend on the form of magnetic circuit. If the magnetic circuit is a toroid in which the thickness of metal is small compared to the diameter of the toroid, the magnetic measurements are easily made, because the magnetic flux density and force are approximately uniform over any cross section. If the test specimen is a long rod whose diameter is small compared with its length, the magnetic measurements for points distant from the ends are likewise readily made. In either case the magnetizing force is calculated from the current-turns of a uniformly wound magnetizing coil. The magnetic flux may be measured in terms of the quantity of electricity which flows through a circuit containing a few test turns wound over the specimen, when the magnetizing force is reversed. It may be measured also by comparing the electromotive forces developed in the test coil and the secondary of a mutual inductance when the magnetizing current and the primary current of the mutual inductance are reversed simultaneously. In practice, however, it is not convenient to have the test specimen in either of the forms just mentioned. Most convenient mechanically is a comparatively short rod of uniform cross section which may be easily machined. If we use a short rod, however, the material under test does not form the whole of the magnetic circuit, and we can no longer assume that the test material comprises the total reluctance of the magnetic circuit. In some cases the greater part of the total reluctance may be in the air path. In a particular case of a cylinder 25 diameters long whose permeability is $3770(=300 \times 4 \pi)$ when the magnetizing force is unity, the reluctance of the air path is I9 times as large as that of the specimen. ${ }^{1}$ In order to reduce the reluctance of the magnetic circuit, the ends of the bar may be connected by a soft iron yoke. With carefully designed and constructed yokes, this gives a very satisfactory magnetic circuit, and as a rough approximation we may assume

${ }^{1}$ Assuming Mann's values for demagnetizing factors. 
that the whole applied magnetomotive force is used to magnetize the test specimens. The reluctance of the yokes must, however, be taken into consideration. This may be done in two ways. Using Ewing's double yoke method ${ }^{2}$ with two different lengths between yokes of the test specimens and magnetizing coils, one can eliminate the reluctance of the yokes, assuming it to be the same in each case for corresponding values of the induction, and so obtain the true induction curve.

A second method of obtaining data free from errors due to the yokes is to apply to the yokes and joints a compensating magnetomotive force which shall overcome the reluctance of these parts of the magnetic circuit. When properly compensated, all parts of the magnetic circuit are at the same magnetic potential and consequently there is no magnetic leakage from one part of the circuit to another. This idea of a distributed and adjustable magnetomotive force was suggested to me by Prof. E. B. Rosa about two years ago. I am indebted to him not only for the original suggestion but also for his hearty cooperation throughout the course of the investigation. The theory of this compensating magnetomotive force is made clear from a consideration of the magnetic condition of a straight bar surrounded by a magnetizing solenoid.

\section{THEORY OF A DISTRIBUTED MAGNETOMOTIVE FORCE.}

In order that there shall be no magnetic leaking anywhere in the rod, it is necessary that the magnetic potential of the rod be everywhere the same. That is, that in the infinite rod the rise of magnetic potential in each element of length due to the current must be exactly equal to the fall of magnetic potential in that element due to the reluctance of the iron. If the iron is of uniform permeability and constant cross section, and the ampere turns on the solenoid be uniform throughout the length, then the rod will be of uniform magnetic potential.

If, however, between $A$ and $B$ (Fig. I) the iron is of lower permeability than the average or its section is a little less, the fall of magnetic potential will be a little greater between $A$ and $B$ than 
otherwise, and $B$ will be at a lower magnetic potential than $A$. The result is a leakage of lines from $A$ to $B$. On the other hand, if the iron between $A$ to $B$ has a higher permeability than elsewhere, or has a slightly larger section, then the fall of potential from $A$ to $B$ will be less than otherwise, and the magnetic potential at $B$ will be higher than at $A$ and the leakage will be in the reverse direction from $B^{\prime}$ to $A^{\prime}$. . In like manner, if the iron is perfectly uniform in section and permeability, but the winding of the magnetizing coil is a little more open between $A$ and $B$ than elsewhere, so that there is a slightly less number of current-turns per $\mathrm{cm}$ there than elsewhere, then $B$ will be at a lower magnetic potential than $A$ and a leakage will occur from $A$ to $B$. If, however, the wire is wound closer, then $B$ will be at a higher magnetic potential and the leakage is reversed from $B^{\prime}$ to $A^{\prime}$. Thus,

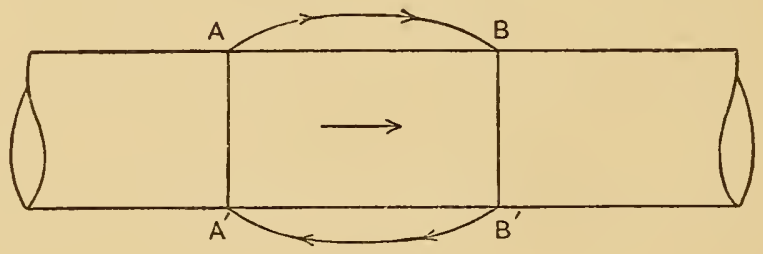

Fig. 1.

in general, in such a magnetic circuit, or in any magnetic circuit, in order that there shall be no magnetic leakage the magnetomotive force due to every element of winding must be just sufficient to overcome the reluctance of that element of the magnetic path within the winding. This requires in practice extreme care in the preparation of the magnetizing coils for the case of uniform rods, and very nice adjustment of the magnetizing windings in the case of nonuniform circuits, which are, of course, what we generally have.

Fig. 2 illustrates this distribution of magnetomotive force. We have here a uniform straight rod with its ends joined by a yoke of low magnetic reluctance, and magnetizing turns wound uniformly over the rod and distributed in proportion to the reluctance over the yoke and the joints. We thus secure the same uniformity of induction and magnetizing force for the straight element of the magnetic circuit that we had in the infinitely long rod or in the uniformly wound ring. We may now calculate the magnetic force 
at any point of the straight bar from the magnetomotive force of a unit length of the surrounding solenoid.

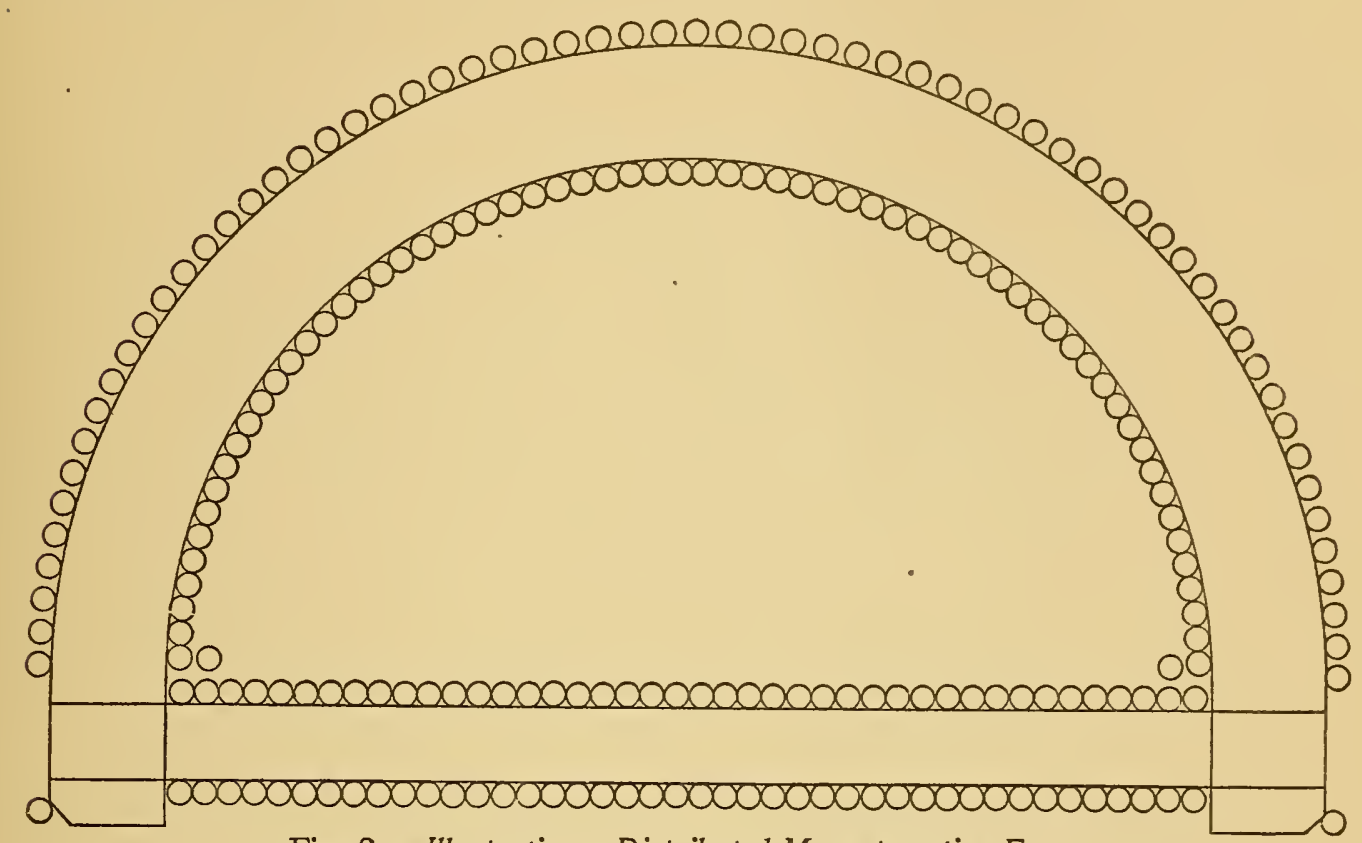

Fig. 2.-Illustrating a Distributed Magnetomotive Force.

The present paper has to do with magnetic circuits of this kind in which the reluctance of various parts of the circuits is overcome by properly placed current-turns.

\section{PRELIMINARY INVESTIGATIONS.}

\section{ON YOKE RELUCTANCE.}

As this method involves the distribution of magnetomotive force in proportion to the reluctance of the magnetic circuit, it is necessary that this reluctance be known. Further, since magnetic reluctance at any part of a magnetic circuit without a proportional magnetomotive force will give rise to magnetic leakage, and leakage is a quantity susceptible of direct measurement while reluctance is not, we shall begin the investigation with a study of magnetic leakage. For this purpose two rods surrounded by two equal and uniform solenoids, joined together at their extremities by heavy soft iron rectangular yokes, formed the magnetic circuit. The joints fit closely, so that the circuit is practically that of the Ewing double yoke $^{2}$ and double bar arrangement. Test coils containing the same number of turns are placed around the middle section of one

${ }^{2}$ Ewing's Magnetic Induction in Iron and Other Metals; 3 d ed., p. 362. 
of the bars and one of the yokes. The bar surrounded by the test coil we shall call the test bar and the other the auxiliary bar. These test coils may be connected so that the electromotive forces developed on reversal of the magnetizing current oppose each other, or they may be used independently. In this way the induction and leakage for various magnetizing forces are readily obtained. Then a single turn of wire is wound around each yoke and connected in series with the main magnetizing coils. With the magnetizing current flowing through these compensating turns and the main solenoids in series, the inductions and leakages are again determined in the same manner as before. This operation is repeated

\section{TABLE I.}

Showing the effect of leakage in the double bar and yoke magnetic circuit.

\begin{tabular}{c|c|c|c|c|c|c|c}
\hline $\mathbf{H}$ & $\begin{array}{c}\text { Uncompen- } \\
\text { sated B } \\
\text { at center }\end{array}$ & Leakage & $\begin{array}{c}\text { Per cent } \\
\text { of leakage. }\end{array}$ & Leakage $\div \mathrm{H}$ & $\begin{array}{c}\text { Number of } \\
\text { turns to } \\
\text { compensate }\end{array}$ & $\begin{array}{c}\text { Column (5) } \\
\begin{array}{c}\mathbf{4} \\
\text { Colum (6) }\end{array}\end{array}$ & $\begin{array}{c}\text { Reluctance of } \\
\text { Yoke in Terms } \\
\text { of equivalent } \\
\text { Lenth of } \\
\text { Test Bar }\end{array}$ \\
\hline 10 & 13000 & 440 & 2.9 & 44 & 5.8 & 7.6 & 5.8 \\
20 & 14680 & 620 & 4.2 & 31 & 4.3 & 7.2 & 4.3 \\
30 & 15580 & 780 & 5.0 & 26 & 3.6 & 7.2 & 3.6 \\
40 & 16200 & 820 & 5.1 & 20 & 3.0 & 6.7 & 3.0 \\
50 & 16670 & 830 & 4.9 & 17 & 2.5 & 6.8 & 2.5 \\
\hline
\end{tabular}

Constants of apparatus:

Length of rods, $27 \mathrm{~cm}$.

Length between yokes, $12.85 \mathrm{~cm}$.

Section of rods, $.277 \mathrm{~cm}^{2}$

Length of yoke between rods, $2 \mathrm{~cm}$.

Section of yokes, I. $5 \times 1.5=2.25 \mathrm{~cm}^{2}$

Primary turns on each rod $=100$.

Secondary turns, 50 on bar and 50 on yoke.

The number of turns to compensate is determined by interpolation in Fig. 3. The equivalent reluctance of yoke (column 8) is computed by dividing the total magnetomotive force applied to one yoke by $H$. It therefore expresses the length of rod to which the yoke is equivalent. 
with other numbers of compensating turns about the yokes. The results are shown in Table I and Fig. 3.

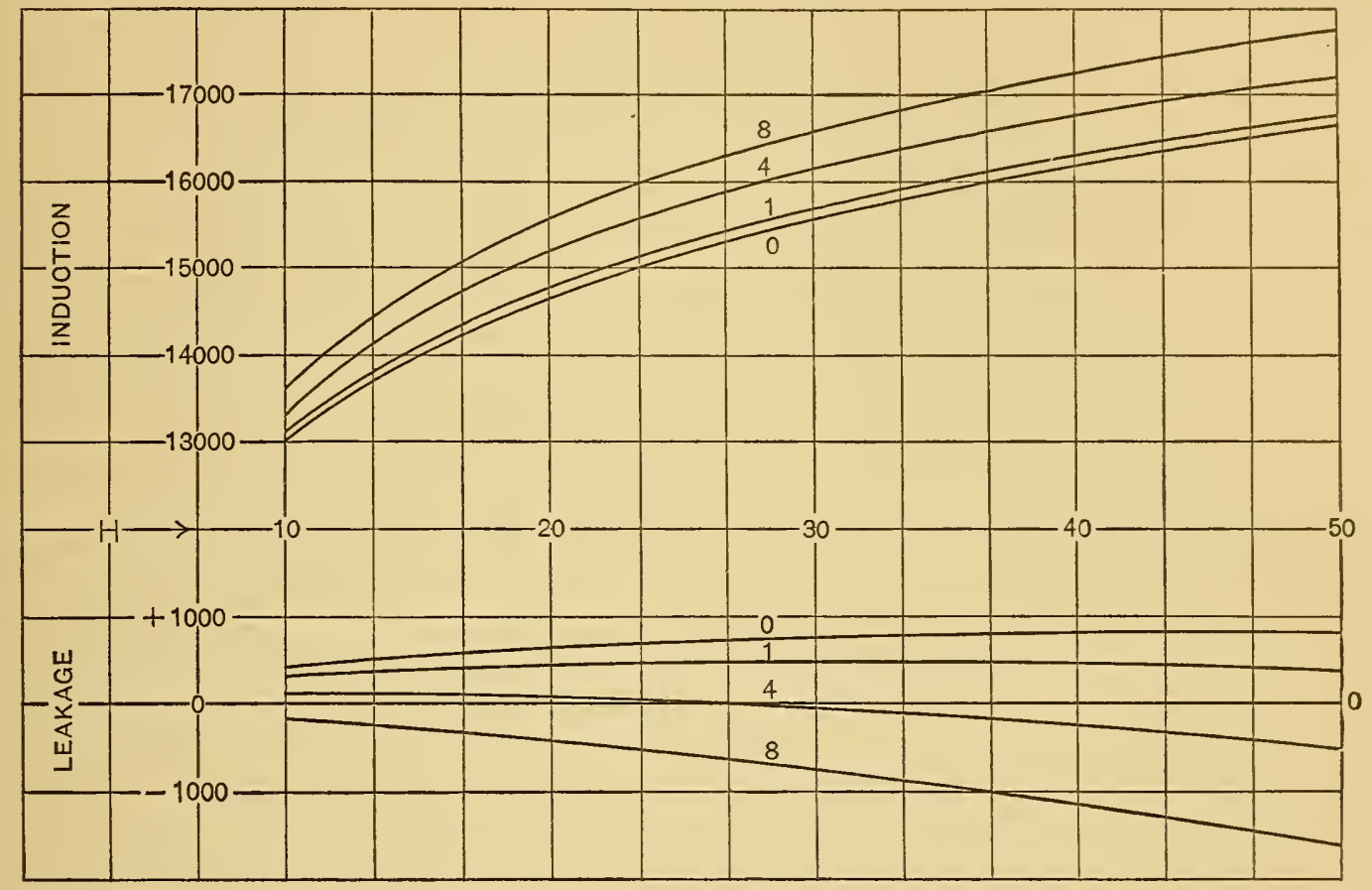

Fig. 3.- Showing the Induction at the Center of Test Rod, and the Leakage between Center of Rod and Center of Yoke for Various Inductions and Compensating Turns wound on the Yokes. (To accompany Table I.)

Curves 0 for no compensating turns.

Curves 1 for one turn on each yoke.

Curves 4 for four turns on each yoke.

Curves 8 for eight turns on each yoke.

Table II shows the data of another experiment carried out with the same purpose as the preceding, but from a different point of view. In this set-up two pairs of long rods of similar material are used with two different sets of magnetizing coils whose lengths are in the ratio of $2: \mathrm{x}$, but whose other dimensions are equal. Test coils are placed one on the middle of the bar and one distributed with one-half over each end of the bar. Distributing the test coil tends to eliminate any irregularities due to the bar or solenoid. The compensation is effected by coils of 50 turns each placed over the yokes. Through these coils is passed a current which is adjusted independently of the main current.

From these two sets of data and the curve we notice that the leakage between the center of the bar and the center of the yoke, 
and the compensating current-turns necessary to reduce this leakage to zero, are proportional and increase continuously as the magnetizing force increases. Furthermore, the reluctance of the yoke does not bear a fixed ratio to the reluctance of the test piece, but decreases relatively as the magnetizing force increases. Consequently the ratio between the compensating and main magnetizing turns varies, and as seen in the figure a fixed ratio of compensating turns which compensates exactly at some magnetizing force is too small for smaller magnetizing forces and too large for larger ones.

\section{TABLE II.}

Showing the magnitude of the compensation for leakage in the double bar and yoke magnetic circuit at two different lengths.

\begin{tabular}{c|c|c|c|c|c|c}
\hline & & \multicolumn{2}{|c|}{$1=42 \mathrm{~cm}$} & \multicolumn{2}{|c}{$1=21 \mathrm{~cm}$} \\
& B Approximate & $\begin{array}{c}\text { Permeabil- } \\
\text { ity }\end{array}$ & $\begin{array}{c}\text { Compensating } \\
\text { Current }\end{array}$ & $\begin{array}{c}\text { Equivalent } \\
\text { Reluctance } \\
\text { (cm of rod) }\end{array}$ & $\begin{array}{c}\text { Compensating } \\
\text { Current }\end{array}$ & $\begin{array}{c}\text { Equivalent } \\
\text { Reluctance }\end{array}$ \\
\hline 3 & 3110 & 1040 & 0.18 & 3.8 & 0.26 & 5.5 \\
4 & 5370 & 1340 & 0.26 & 4.1 & 0.34 & 5.3 \\
5 & 7240 & 1450 & 0.32 & 4.8 & 0.41 & 5.2 \\
6 & 8800 & 1470 & 0.38 & 4.0 & 0.48 & 5.0 \\
7 & 9750 & 1390 & 0.43 & 3.9 & 0.55 & 5.0 \\
8 & 10750 & 1340 & 0.48 & 3.8 & 0.61 & 4.8 \\
9 & 11400 & 1270 & 0.52 & 3.6 & 0.67 & 4.7 \\
10 & 12080 & 1210 & 0.55 & 3.5 & 0.72 & 4.5 \\
20 & 15010 & 750 & 0.82 & 2.6 & 1.08 & 3.4 \\
40 & 16430 & 410 & 1.12 & 1.8 & 1.44 & 2.2 \\
70 & 17350 & 250 & 1.53 & 1.4 & 1.90 & 1.7 \\
\hline
\end{tabular}

Constants of apparatus:

Length of rod, $45 \mathrm{~cm}$.

Cross section of rod, $.277 \mathrm{~cm}^{2}$.

Lengths between yokes, 42 and $2 \mathrm{I}$.

Yokes same as in Table I.

Test coils 50 turns on middle and 50 turns on end of bar.

Compensating turns on each yoke, 50 .

The equivalent reluctance is obtained by dividing the total magnetomotive force about one yoke by $H$. 
These data are quite in accord with what we might expect from a consideration of the relative values of the reluctances of the test bars and yokes. The yokes having a cross section 9 times that of the bars are worked under a much lower flux density always below that corresponding to the maximum permeability. The reluctance of the yokes therefore decreases as the magnetizing force increases. The reluctance of the test pieces passes through a minimum and then increases continuously. Consequently the reluctance of the yokes will be of less importance at the higher inductions.

\section{FLUX DISTRIBUTION IN THE IRON.}

In the preceding two experiments it has been shown that in a particular case of the double bar and yoke magnetic circuit the leakage between the centers of bar and the yoke amounts to from 2 to 5 per cent, and may be reduced to zero by placing about each yoke current-turns which vary from 5 to 25 per cent of the main magnetomotive force. It now remains to show to what extent the flux density varies in other parts of the magnetic circuit. For this purpose several test coils were distributed over various sections of the magnetic circuit. The magnetomotive force was applied through two solenoids over the rods and two yoke coils over the yokes.

Table III gives data on the distribution of total flux and leakage at different parts of the magnetic circuit for different values of the magnetizing force and for different lengths of rod. These three pairs of rods are of the same lot of low-carbon Bessemer steel, but nevertheless care must be exercised in making comparisons. The three sets of data may not be intercompared too closely, because no correction has been applied for slight changes in the galvanometer constant on different days.

Here we may note:

For any uncompensated system:

The leakage between the center of the bar and the center of the yoke, as well as the leakage between the center and end of bar, is not proportional to the total induction, but passes through a maximum roughly in the region of maximum permeability.

The maximum total induction in the iron occurs through the 
middle section of the test bar for low inductions and near the end of the test bar for higher inductions. This anomaly will be discussed later (see p. 46).

\section{TABLE III.}

Showing magnitude of flux and leakage in the double bar and yoke apparatus, for different values of the main and compensating magnetomotive forces.

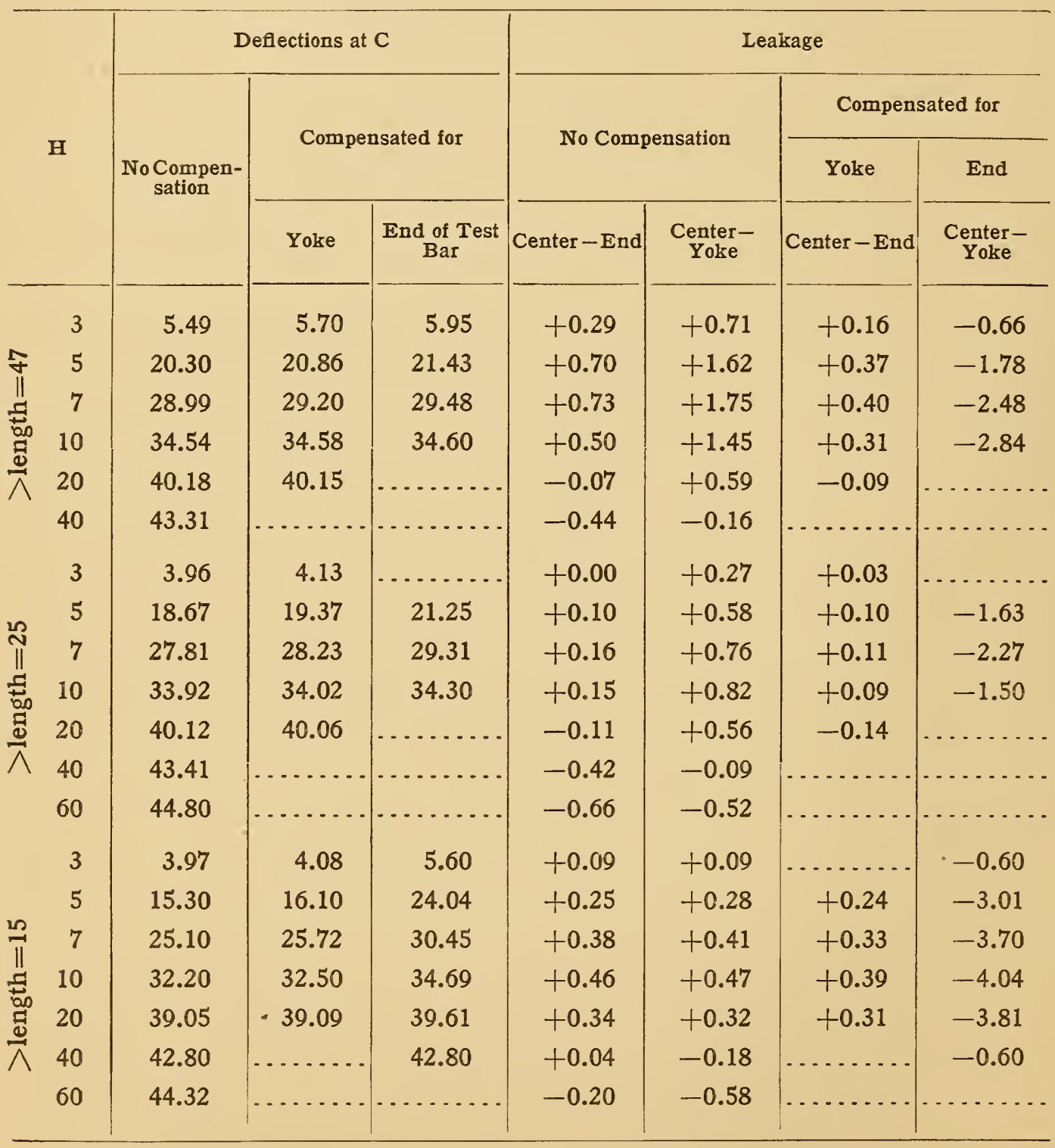

NoTE.-Two test coils are placed over the test bar, one over the middle and one near the end. A third test coil surrounds the yoke. Compensation is secured by adjusting the current in fixed coils about each yoke. 
For the higher inductions, the total flux through the middle section of the bar is less than the total induction through the end of bar, or through the yoke.

In the compensated system:

The change in total induction over the middle section of the bar, due to excitation of compensating turns, is greatest at lower inductions and practically negligible at higher inductions.

At low inductions a much greater induction results if the inductions through sections at the end and middle of the rod are brought to equality by compensation than if sections through the yoke and middle of the bar are used.

In raising the induction at the end of the bar up to that at the middle section a large overcompensation occurs at the yoke. This overcompensation passes through a maximum, and then diminishes rapidly with increase of induction. Other experiments on strips of transformer iron give similar results.

In seeking a solution of the anomalous flux distribution at high flux densities an examination was made of a great variety of magnetic circuits. Figs. 4 and 5 show some of the data thus obtained.

Fig. 4 shows the flux magnitude and distribution along a rod for different conditions of the magnetic return path outside the rod itself. The legend of the figure gives the condition under which the individual flux curves were taken. In this figure we can see how the total induction and the leakage varies with the yoke contact.

The compensated induction (curve 8) is practically constant throughout the length of the rod, and the other curves show, by comparison with it, the total reduction of flux due to the reluctance of the yokes and joints.

Fig. 5 shows the leakage of flux at all points between the center and end of a test specimen $40 \mathrm{~cm}$ long between yokes. The yoke consisted of a three-piece return circuit such as is shown in Fig. Io. Here the yoke effect is very marked, due to the great difference in cross section of specimen and yoke. It is to be observed that the uniformity is greater for $\mathrm{H}=\mathrm{I}$ and 3 than it is for $\mathrm{H}=2$, so that a maximum nonuniformity occurs in the region of maximum permeability of the test piece. 


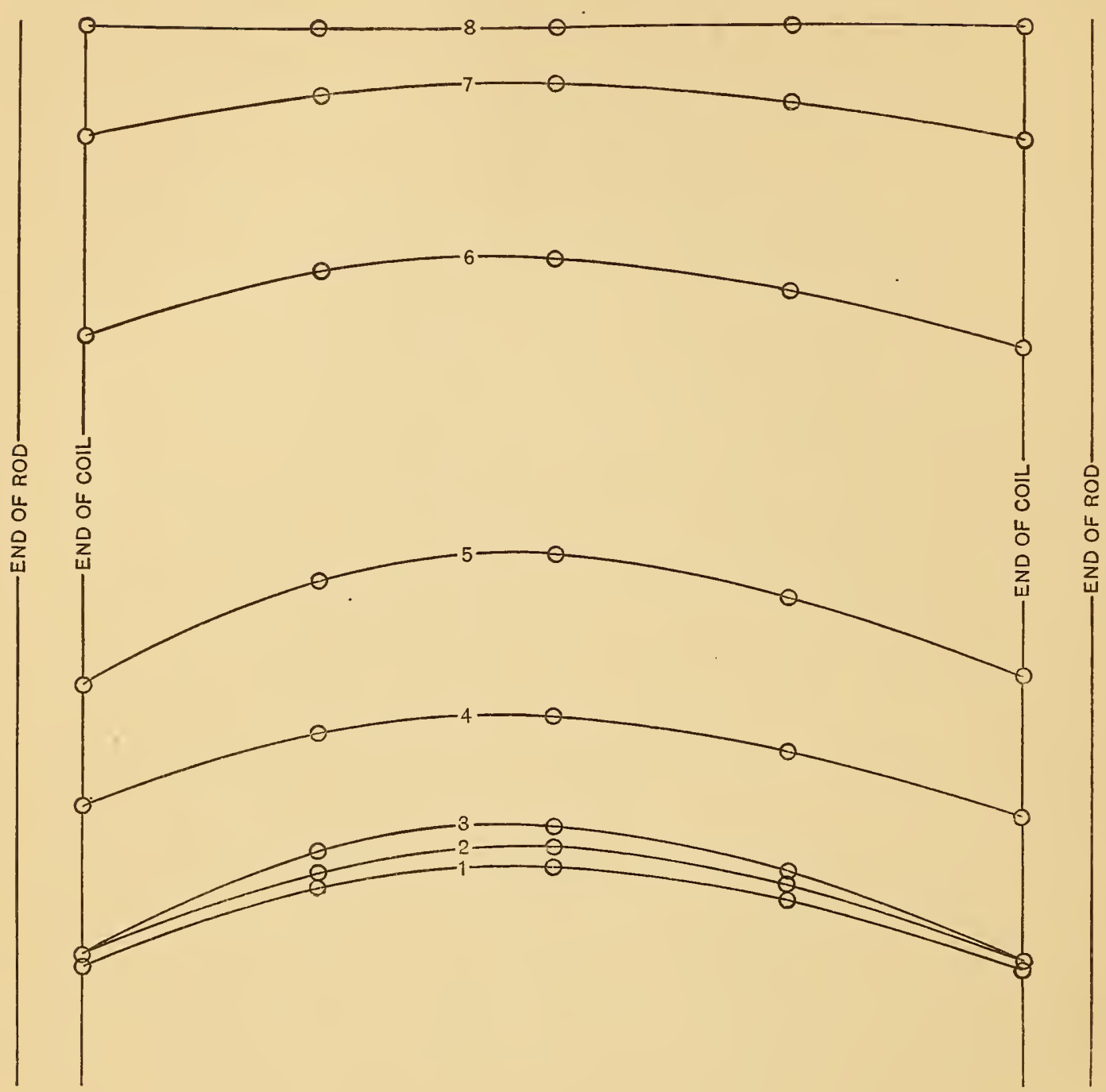

Fig. 4.-Showing the Magnitude and Distribution of Flux along the Length of a Rod $47 \mathrm{~cm}$ Long under Varying Conditions. Main Magnetizing Current Constant.

Curves 1-4 were taken with a second similar bar placed parallel to the first, and $6.5 \mathrm{~cm}$ distant.

Curve 2: Test bar alone is magnetized.

Curve 1: Both bars magnetized, with like poles adjacent.

Curve 3: Both bars magnetized, with unlike poles adjacent.

Curve 4: Same as 3, but with massive yokes buttıng against ends.

Curve 5: Same as 4, but with rods $2 \mathrm{~cm}$ apart.

Curve 7: Same as 6, but with better fitting rectangular yokes.

Curve 6: Same as 5, but with rods clamped in yokes.

Curve 8: Same as 7, but with compensating turns on yokes. 
In each curve the leakage is less near the yoke than a little farther away, thus seeming to indicate the presence of a magnetomotive force in the yoke itself.

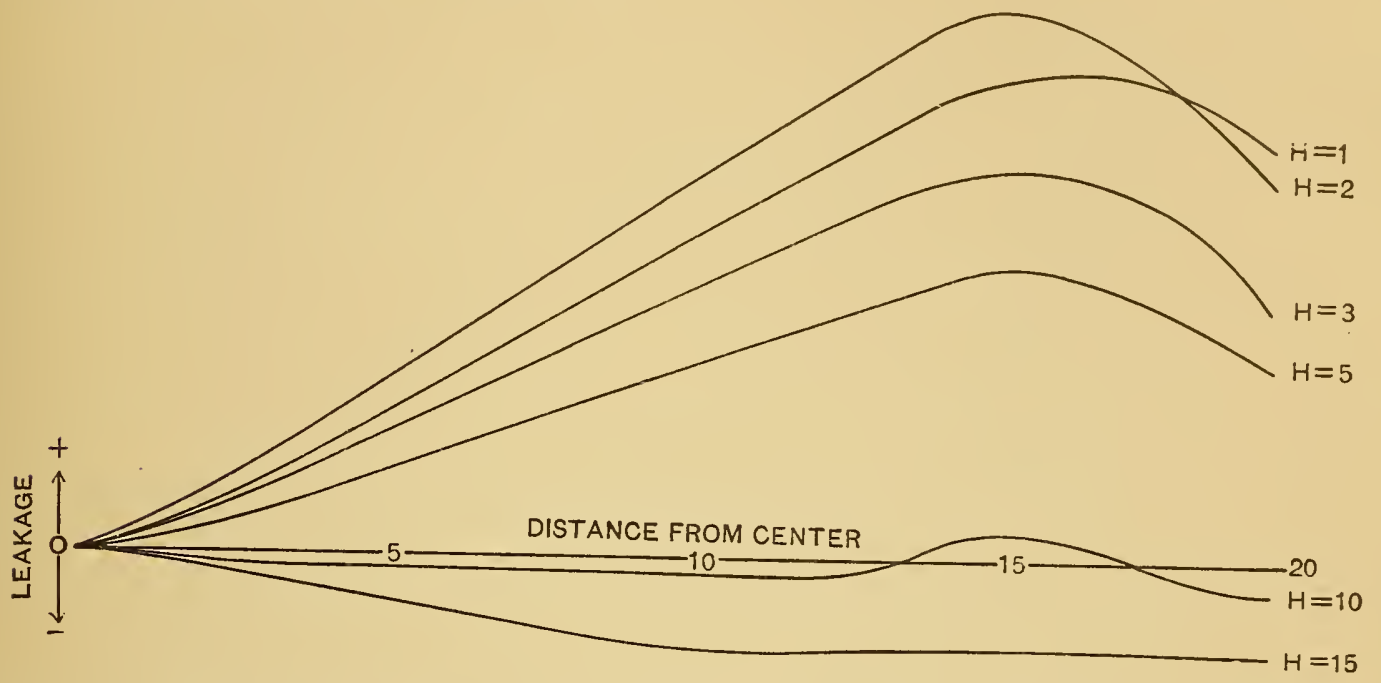

Fig. 5.-Showing Leakage under Various Magnetizing Forces in a Thin Strip of Transformer Iron Clamped in a 3-piece U-shaped Yoke.

For the higher magnetizing forces this magnetomotive force predominates and the middle section of specimen is a minimum for the total flux.

\section{LEAKAGE FLUX IN AIR.}

Up to this point the leakage has been studied as a loss of flux from the bar into the air. The distribution of this leakage in the air is of interest and was investigated by means of a small magnetic needle. In order that the needle might not disturb the field appreciably, it was made as small as it could be conveniently. It consisted of $3 \mathrm{~mm}$ of steel broken from the pointed end of a small sewing needle, and was suspended by a single cocoon fiber.

Fig. $6,(a),(b)$, and $(c)$, shows by the arrowheads the direction of the magnetic field in air. The small arrowheads separated by dashes show the direction of flux in the iron. The circles indicate uncertainty in the direction of field. In (a) no compensating current was used, but in $(b)$ and $(c)$ a current was passed through fixed turns about the yoke till the flux was the same at the center and ends of bar. (b) and (c) differ only in the direction of magnetizing forces and leakage. Fig. $6(d)$ shows more fully the flux in the air 

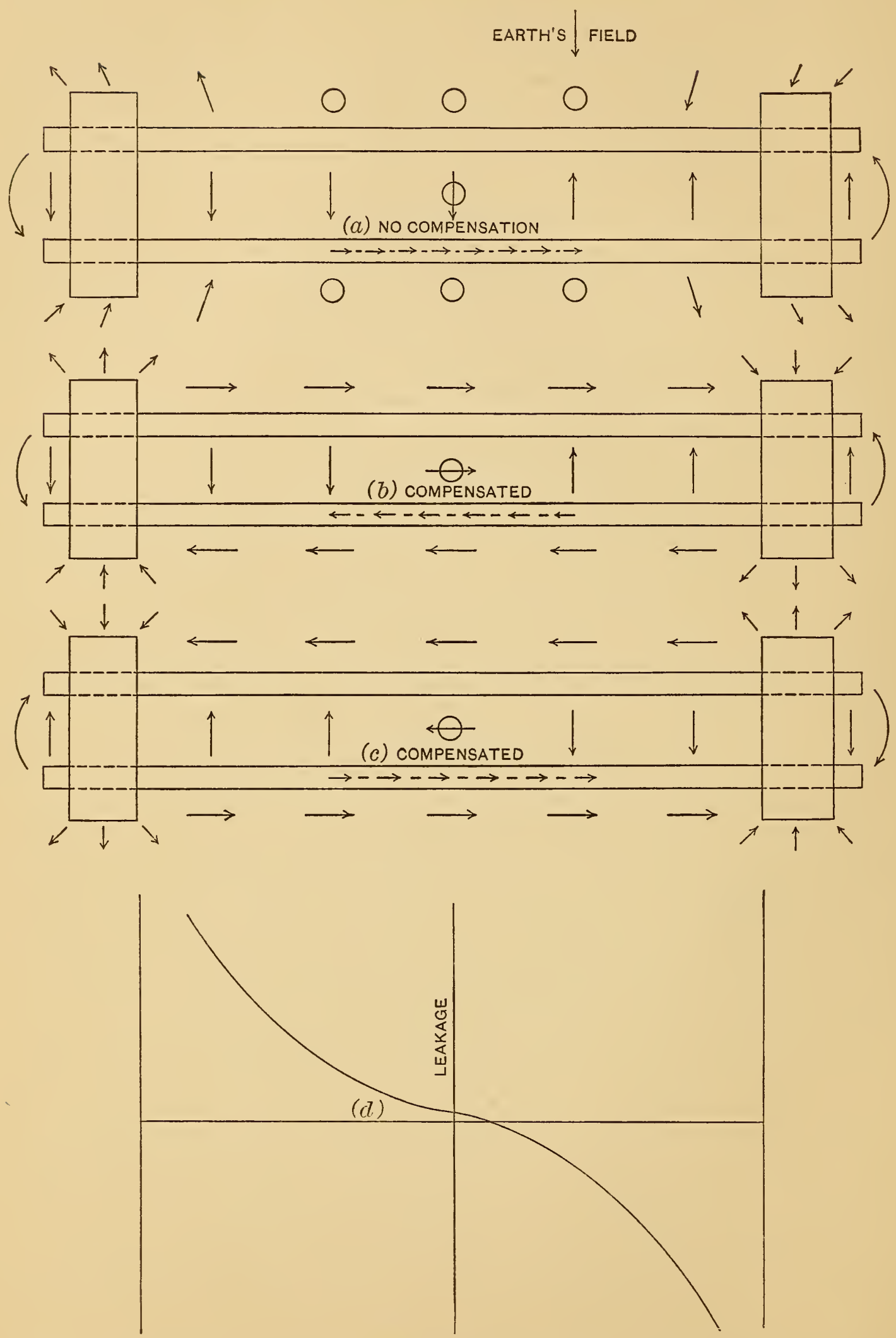

Fig. 6.- Showing the Distribution of Leakage in the Double Bar and Yoke Apparatus, both with and without Compensating Turns wound on the Yokes. Equal Solenoids over each Rod. 
space between the two specimens. To obtain this last diagram, a test coil of 50 turns, $2.5 \mathrm{~cm}$ in diameter, was held at various positions while the magnetizing current was reversed and the ballistic throw noted. From these figures we observe that with no compensating magnetomotive force the external field is similar to that of two magnets placed with unlike poles together. The yokes act merely as enlargements of the ends of the test bars. The field outside the rectangle is weak and the pole length somewhat less than the distance between the yokes. When compensated by coils placed on the yokes so that the end of test rod carries the same flux as the middle section, the direction of field between the bars is reversed, while the field outside the rectangle and near the rods is strongly defined and in the same direction as the induction within the bars. In general, the external field is due to magnetization of the yokes by the compensating current.

It is evident that the field at the middle section of the test specimen is greater than that due to the magnetizing solenoid alone, and that this method of compensating is not free from objection. It is also evident that a magnetic needle is not a reliable indicator of the uniformity of flux within the test specimen.

\section{REACTIVE FORCE OF THE YOKES.}

From the preceding it is evident that the yokes do have an appreciable influence on the distribution of flux within the bar. This is further evidenced by a consideration of Fig. 7, which represents a portion of a magnetic circuit. When the magnetizing current is flowing the magnetic flux has the direction indicated by the arrows. The yoke is here magnetized to an intensity which varies in magnitude from point to point, but has the same general direction as indicated by the arrows. It is shown in works on the theory of magnetism ${ }^{3}$ that such a magnetized system creates a field which at any point in space has the value

$$
H_{i}=\Delta \int d v\left(I \Delta \frac{\mathrm{I}}{r}\right)
$$

where $I=k H=$ intensity of magnetization.

${ }^{3}$ Abraham: Theorie der Elektrizität, vol. I, p. 228, 3d ed. 2 I $92-$ No. $\mathrm{I}-\mathrm{O} 9-4$ 
Applying this equation to the system of Fig. 7 we see that the force due to the yokes has a component of the same sign as the impressed force for all points within the solenoid, which increases with the section of the coil, the magnitude of the impressed magnetizing force, and the susceptibility of the yoke, and decreases with increase in the section of the specimen and the distance from the yoke. These same conclusions are reached by supposing the faces of the yokes opposite the ends of the solenoids to be covered with free magnetism of the signs indicated in the figure.

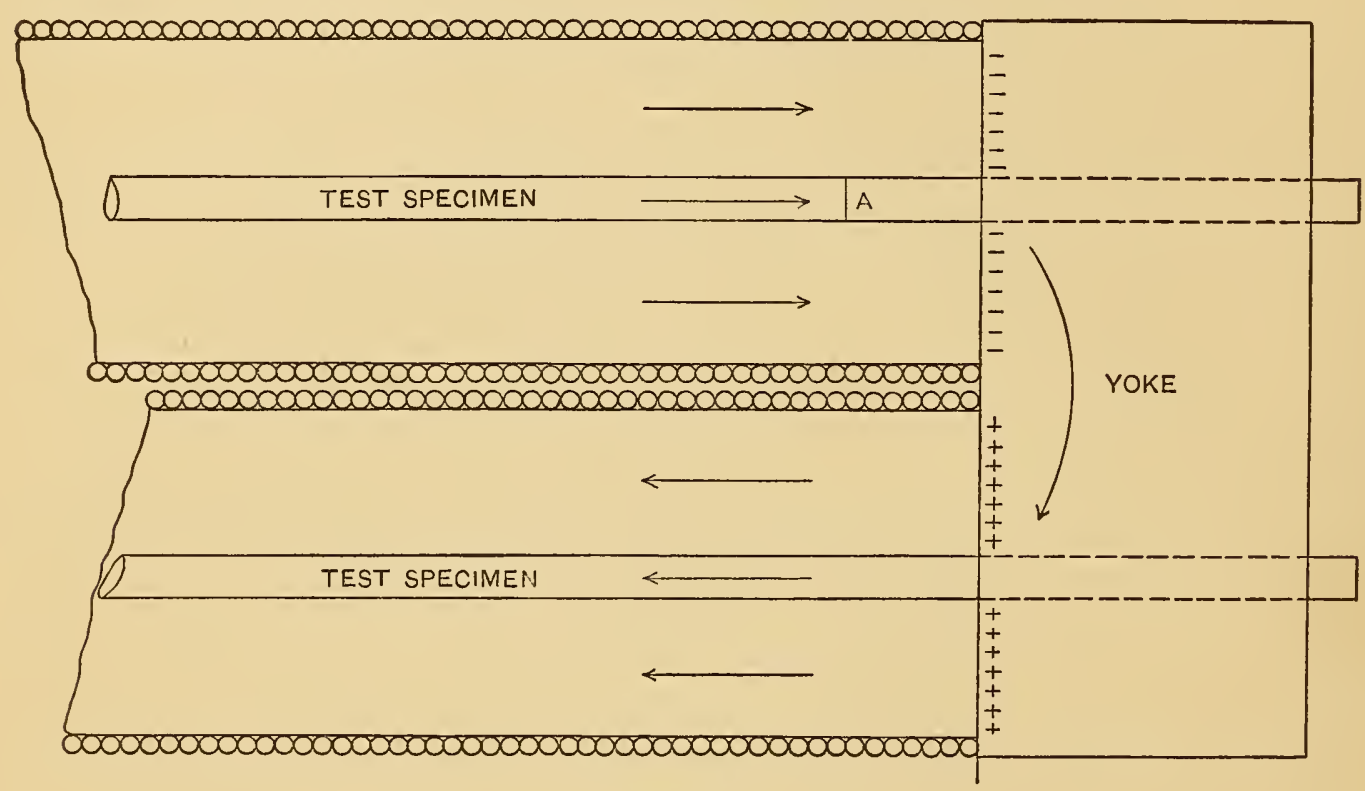

Fig. 7.

The resultant magnetic force is therefore composed of two partsthat due to the solenoid alone, which diminishes as the yokes are approached, and that due to the magnetization of the yokes, which increases as the yokes are approached. It would seem therefore possible, by varying the conditions, to make the force near the end of the specimen either greater or less than the force at the center. The anomalous flux distribution may therefore be accounted for by supposing that the yoke effect predominates. To settle the matter beyond doubt, other experiments using test specimens and yokes of various cross sections were made. In these experiments the test coils were wound close upon the test pieces and the magnetizing coils were free from any appreciable irregularity. To eliminate any effect due to differences in the two test pieces, the current through each solenoid was adjusted so that 


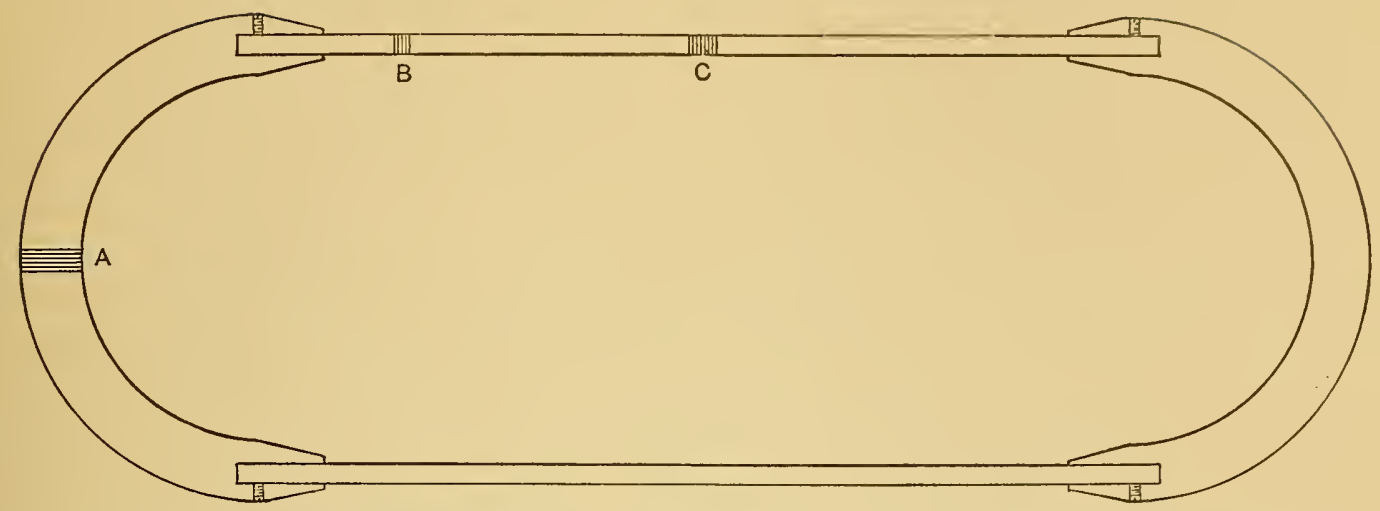

Fig. 8. - Showing the Arrangement of Magnetic Circuit and Test Coils as used in determining the Data of Tables IV and $V$. The Magnetizing Coils consist of a Uniform Solenoid over each Bar, Uniform Windings over the Yokes, and Concentrated Windings over the Joints. Test Coils are placed at $A, B$, and $C$.

TABLE IV.

Showing the flux and leakage distributions for double bar and yoke apparatus using curved yokes as shown in Fig. 8 (length between yokes $=$ $40 \mathrm{~cm}$ ).

\begin{tabular}{|c|c|c|c|c|c|c|c|}
\hline \multirow{3}{*}{$\mathbf{H}$} & \multicolumn{3}{|c|}{ Deflections } & \multicolumn{4}{|c|}{ Leakage } \\
\hline & \multirow{2}{*}{$\begin{array}{c}\text { No Compen- } \\
\text { sation }\end{array}$} & \multicolumn{2}{|c|}{ Compensated for } & \multicolumn{2}{|c|}{ No Compensation } & \multicolumn{2}{|c|}{ Compensated for } \\
\hline & & Yoke $\mathrm{C}=\mathrm{A}$ & End $C=B$ & $\mathrm{C}-\mathrm{B}$ & $\mathrm{C}-\mathrm{A}$ & Yoke C-B & End C-A \\
\hline 1 & 0.57 & 0.57 & 0.57 & 0.03 & 0.18 & 0.00 & 0.00 \\
\hline 2 & 1.10 & 1.14 & 1.14 & 0.06 & 0.29 & 0.00 & 0.00 \\
\hline 3 & 5.86 & 7.16 & 6.76 & 0.31 & 1.25 & -0.15 & +0.39 \\
\hline 4 & 12.28 & 13.94 & 13.75 & 0.48 & 1.76 & -0.05 & +0.34 \\
\hline 5 & 18.41 & 19.92 & 20.31 & 0.76 & 1.92 & +0.24 & -0.51 \\
\hline 6 & 23.61 & 24.72 & 25.31 & 1.04 & 2.08 & +0.42 & -1.12 \\
\hline 7 & 27.30 & 28.14 & 28.69 & 1.22 & 2.22 & +0.55 & -1.43 \\
\hline 8 & 30.27 & 30.83 & 31.18 & 1.29 & 2.34 & +0.57 & -1.47 \\
\hline 9 & 32.27 & 32.68 & 32.98 & 1.21 & 2.25 & +0.50 & -1.48 \\
\hline 10 & 34.01 & 34.19 & 34.33 & 1.11 & 2.19 & +0.48 & -1.54 \\
\hline 20 & 40.26 & 40.21 & 40.26 & 0.29 & 1.23 & +0.14 & -0.71 \\
\hline 40 & 43.84 & 43.80 & 43.80 & 0.02 & 0.60 & 0.00 & 0.00 \\
\hline 70 & 45.63 & & 45.63 & 0.00 & 0.16 & & +0.16 \\
\hline
\end{tabular}

Constants of apparatus as shown in Fig. 8. 
the flux densities in the two specimens were equal. The investigation showed that for specimens of much smaller section than the solenoid, the excess of the flux density of the middle section over that of a section nearer the end decreased as the yokes of larger size were used, and finally became negative. For larger specimens, which filled the solenoids, this reversal did not occur.

\section{CURVED YOKES.}

With a view to keeping a greater proportion of the flux within the iron and reducing the disturbing leakage fields, curved yokes such as shown in Fig. 8 were designed. These yokes have the advantage of producing a flux which as it leaves the yoke has the same direction as the flux developed in the test rods. Furthermore, it is easy to so distribute magnetizing turns over the yoke that the sections of greater reluctance have a greater magnetizing force. In these yokes the winding over the main part of the yoke is uniform, while over each nose it is more closely wound. As before, the compensating current is adjusted independently of the main current. Table IV shows some data taken with this apparatus, using rods $40 \mathrm{~cm}$ long between the yokes.

With no compensating current the flux is a maximum at the middle of the test rod and a minimum at the middle of yoke. The anomalous flux distribution along the test rod, which was noted in the rectangular yokes, has here disappeared. When the compensation is adjusted so that the middle and end sections of the test bar are crossed by the same total flux, there is an overcompensation as far as the flux in the yoke is concerned. This gives rise to a field at the middle of specimen, in addition to that developed by the coil.

Table $\mathrm{V}$ shows the magnitude of the compensating currents required in the preceding experiment. Notice here that the compensating current does not increase continuously, but passes through a maximum for each form of compensation. The equivalent length of the yoke passes through a maximum, and at the highest magnetizing force used has the extremely low value of I mm. Experiments on other lengths of specimen give results substantially in accord with the preceding. 
TABLE V.

Showing the compensation required and the equivalent reluctance of the curved yokes.

\begin{tabular}{c|c|c|c|c}
\hline \multirow{2}{*}{$\mathrm{H}$} & \multicolumn{3}{|c|}{ Compensating Current } & $\begin{array}{c}\text { Equivalent Length } \\
\text { of Yoke }\end{array}$ \\
\cline { 2 - 3 } 1 & When C=A & When C=B & Sum & \\
\hline 2 & 0.010 & 0.010 & 0.020 & 1.31 \\
3 & 0.020 & 0.020 & 0.040 & 1.31 \\
4 & 0.081 & 0.057 & 0.138 & 3.0 \\
5 & 0.096 & 0.084 & 0.180 & 2.9 \\
6 & 1.07 & 1.38 & 2.45 & 3.2 \\
7 & 1.13 & 1.85 & 3.08 & 3.5 \\
8 & 1.25 & 2.12 & 3.37 & 3.1 \\
9 & 1.29 & 2.19 & 3.48 & 2.6 \\
10 & 1.30 & 2.21 & 3.51 & 2.6 \\
20 & 1.23 & 2.23 & 3.46 & 2.3 \\
40 & 0.80 & 0.92 & 1.72 & 0.6 \\
& 0.34 & 0.00 & 0.34 & 0.1 \\
\hline
\end{tabular}

Fig. 9 shows the distribution of flux along one-fourth the length of the magnetic circuit, consisting of two rods and two curved yokes. The circles show where measurements were taken. In each curve there are two circles containing crosses indicating that at these two points the fluxes are equal. Curve 2 of this figure shows the normal flux distribution under the action of the main magnetizing coils alone. The other curves were obtained by adjusting to equality the fluxes at the two sections indicated. Here we observe that when the system is magnetized by the main solenoids and no compensation is used, the maximum of flux (in this particular case) occurs between the center and the end of test rod. To secure equality of flux across the middle section and any other section between it and the section of maximum flux it is necessary to reverse the current in the coils surrounding the yokes. The resulting indications at all points are lowered and the nonuniformity throughout the magnetic circuit beyond the sections of compensation is increased. To secure equality of flux across the middle section and any section beyond the section of maximum flux, direct compensation is applied. As a result the total 
induction at all points is raised and the uniformity is improved. A comparison between the dotted lines representing the induction under full compensation and the other curves shows the relative magnitude of the true and apparent inductions under the various conditions.

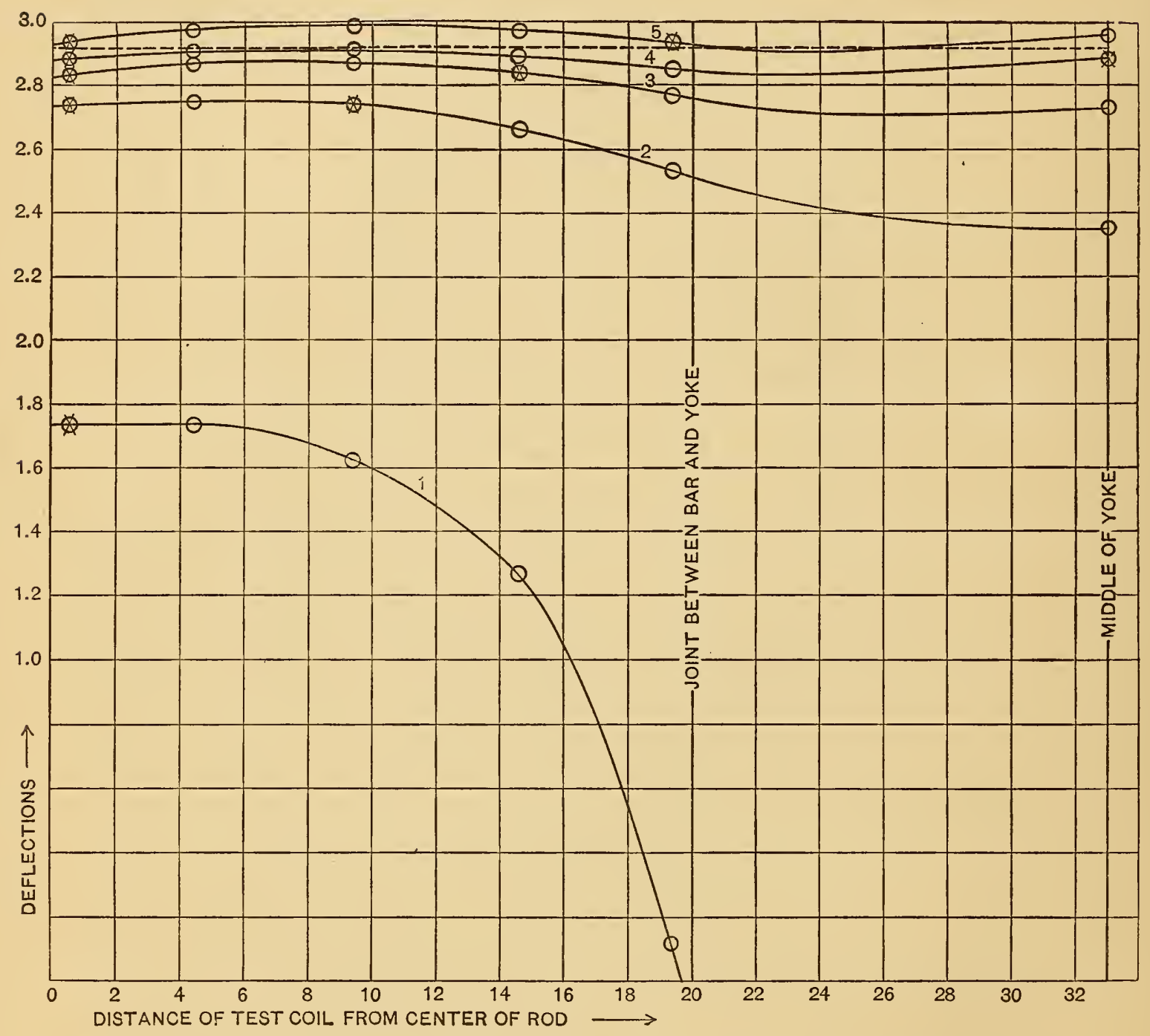

Fig. 9.-Showing the Distribution of Flux along a Double Yoke and Rod Magnetic Circuit with Various Degrees of Compensation. The Crosses on each Curve indicate the two Cross Sections, the Fluxes through which are made equal. The Solid Curves are for Compensation on the Yokes only. The Straight Dotted Line is, for Compensation on the Yokes and over the Joints, Adjusted Separately.

\section{DOUBLE COMPENSATION.}

From what has preceded, it is quite obvious that it is impossible to secure the same flux across every cross section of a ferromagnetic circuit by means of a main magnetizing current and a single 
compensating current of the same relative distribution for all inductions. To secure better uniformity of flux, the compensating turns were divided, into two sections and the current through each portion was adjusted separately. With one compensating coil wound over the yoke and a second wound over the test piece as close to the joint as possible, a quite uniform flux was secured throughout the length of the rod. If the rods were of identical magnetic properties, the compensating turns about the joints gave results practically as good as the double compensation. The difference in permeability between the two rods which are supposed to be alike (i. e., cut from the same stock length or from adjoining portions of the same sheet) and the fact that the bars constitute the greater portion of the reluctance of the circuit, may cause as much trouble in compensating as the total reluctance of the yoke. For this reason it was found desirable to divide the total applied magnetomotive force into three sections: (I) a uniform solenoid over the test piece, (2) a similar uniform solenoid over the auxiliary bar, and (3) a set of four short coils wound over the ends of the rods and connected in series. These three components of the total magnetomotive force are capable of independent adjustment. The currents are adjusted until the fluxes across the middle sections of the test and auxiliary bars and across the end sections of the test bar are equal. When these adjustments have been made it is found that the flux through the yokes does not differ materially from uniformity and the change in induction at the center of the test piece when this outstanding nonuniformity is compensated for is inappreciable. From a practical standpoint, it is too laborious to adjust more than three independent currents in securing uniformity of flux. With two adjustable currents we have seen that the flux is not sufficiently uniform when the magnetizing coils about the test and auxiliary rods are in series and adjusted as a unit, while a compensating current about the yokes is adjusted separately. Other experiments, not recorded, show that a little better uniformity is secured if the compensating coils are placed over the ends of the rods as near as possible to the joints instead of being wound over the yokes. With three adjustable currents, we may have the two main magnetizing coils in series for one adjustment and yoke and joint coils for the other two adjustments, or we may adjust the 
two main magnetizing coils independently and make the third adjustment on a joint coil. The latter gives better satisfaction than having the third adjustable coil wound over the yoke.

\section{VARIOUS FORMS OF YOKES.}

When the magnetizing currents about the two rods are adjusted separately, it becomes immaterial whether the rods are of the same magnetic properties. In fact, they are seldom of the same properties; hence the necessity of the separate adjustment. We are thus independent of the relative properties of the test and auxiliary rods. We may therefore replace the auxiliary rod by something more convenient. This was done in a variety of ways.
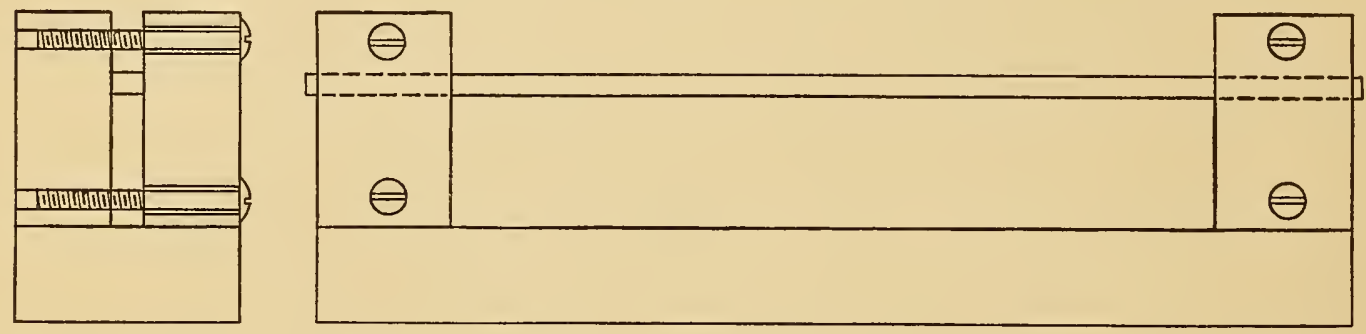

Fig. 10.-Showing a Magnetic Circuit in which the Ends of the Test Piece are Clamped in Two Small Yokes which in turn are Clamped to a Massive Soft Iron Base.

Figs. IO, II, and I 2 show three forms that were examined quite carefully. Fig. Io is modified from the double yoke and bar apparatus by replacing the auxiliary rod by a massive bar faced off on its upper surface. A set of ordinary double yokes are faced off on one end so as to form a close joint with the yoke base. The test bar is clamped between the yokes in the usual manner. Magnetizing turns were distributed over the yoke base and horns, roughly proportional to the reluctance. This was not difficult, as they were made from the same large bar of Norway iron. A second compensating magnetizing coil was wound around the end of the specimen. Test coils for the adjustment of the compensation were placed over the middle and end of bar and middle of yoke base. This arrangement gave fair satisfaction, and was adjustable to any length of rod. Knowing that the projecting corners of the yoke horns are the sources of disturbing fields, the yoke horns were modified to the form shown in Fig. II. Here the joint between base and horn is improved by increasing the surface. The jaws within which the specimen is clamped project 
over the specimen in such a way as to permit the winding of compensating turns concentrically over yoke and rod. This tends to
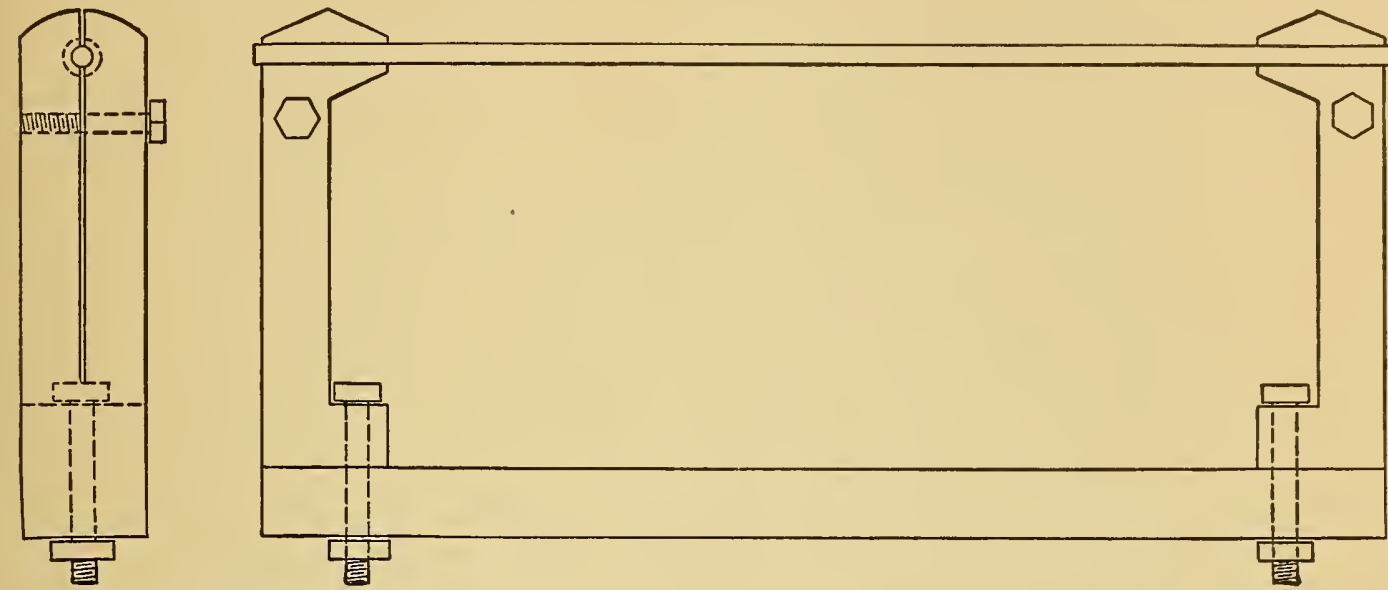

Fig. 11.-A Modification of the Yokes of Fig. 10 in which the Objectionable Corners have been Removed.

reduce the leakage field through the air. A third form is shown in Fig. I 2. Here the yoke consists of two equal rectangles, with rounded corners, placed one over the other. Between these two yokes and along the line of the greater diameter is clamped the specimen. This apparatus is confined to specimens of one length only, but has the advantage of a more uniform yoke, unbroken transversely.

While these forms of magnetic circuit were all fairly satisfactory, they do not offer any marked advantage over the simple double bar and yoke circuit. Many forms of small yokes were tried. Here the aim was to improve the magnetic contact and to lessen the disturbing field due to local poles on the yokes.

Fig. $\mathrm{I} 3$ is one of a pair of the ordinary small yokes, such as used by Ewing. Here the contact is made by pressing the test bar against the walls of the yoke holes. Usually the rod is a trifle smaller in diameter than the hole which receives it, and the result is that the thumbscrew in forcing a good contact on one side of the rod destroys the contact on the other. Fig. I4 illustrates this point nicely. To reduce this trouble, yokes of the form of Fig. I 5 were made. These yokes are in two pieces, and fitted together so that heavy machine screws draw the parts together. The bar comes in contact with the yoke along two surfaces, and the air space is less with these yokes than with the preceding, when made with the same care. A very good contact was obtained with yokes 

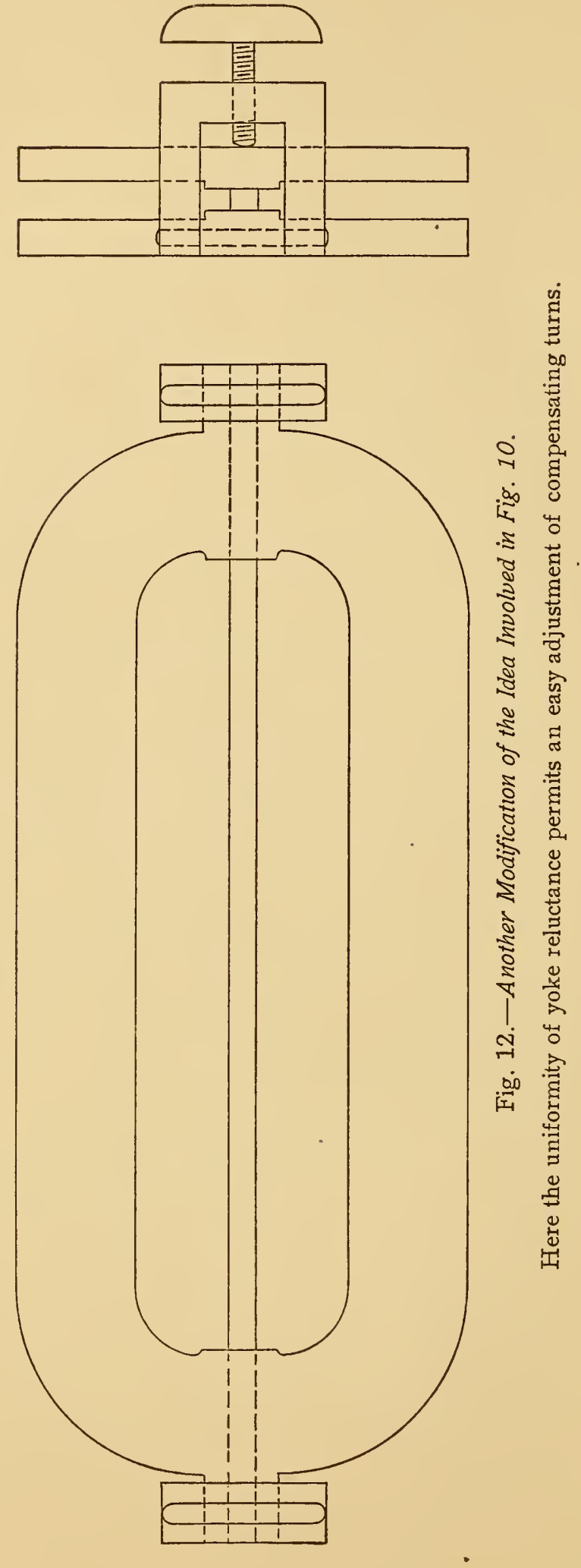


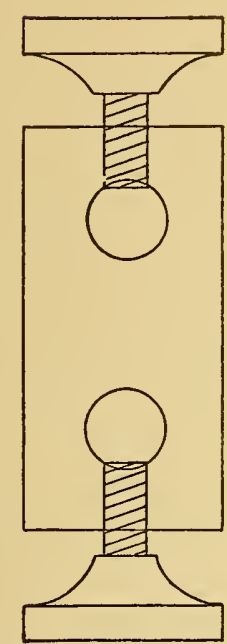

Fig. $13 .-$ Small Ewing Yoke (to be u s ed in pairs).

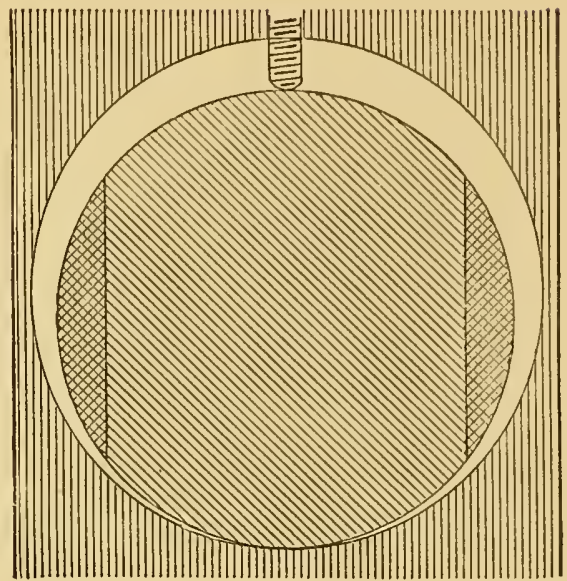

Fig. 14.-Showing the Contact of a Round Rod in a Round Hole, and held in Place by a set Screw. This Form is used in the Ewing Double Yoke, Ewing Bridge, etc. The Double Cross Hatching shows the Portion that may be Removed to Form Flat Surfaces for Parallel-jawed Yokes.

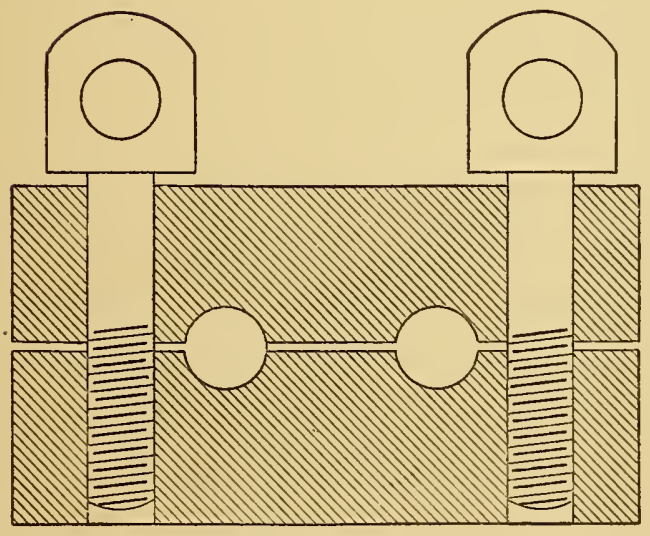

Fig. 15.

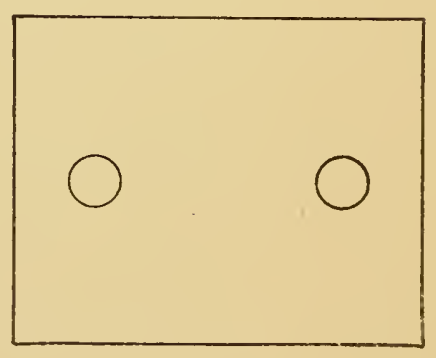

Fig. 16.

Modifications of Ewing Small Yoke to secure better Magnetic Contact. 
of the form of Fig. I6. The holes of this set of yokes were made to fit a particular set of rods, and were very carefully machined to a driving fit. No set screws were used. Of course this is not practical for general use. Good contact in the curved yokes used in the set-up of Fig. 8 is secured by having the bottom and sides of the hole carefully machined. The rod, too, if necessary, is dressed off on the end. We thus have a contact on end and sides. In order to avoid denting the specimen, the set screw does not work directly against the specimen, but is separated from it by a small iron disk. This, from the purely magnetic standpoint, is the most satisfactory yoke used. It requires, however, a test specimen of a definite diameter and length. This is a practical objection.

In an effort to make the reluctance of the whole circuit as uniform as possible, semicircular yokes were made, having the same cross section as the test piece. These yokes did not offer any advantages to warrant their use, principally because the contact surfaces were too small.

If a round joint is made to fit any particular size of rod it is necessary to use bushings if a smaller rod is to be tested. In fact, some pieces of commercial apparatus are supplied with bushings for all sizes expected to be used. The use of bushings in that part

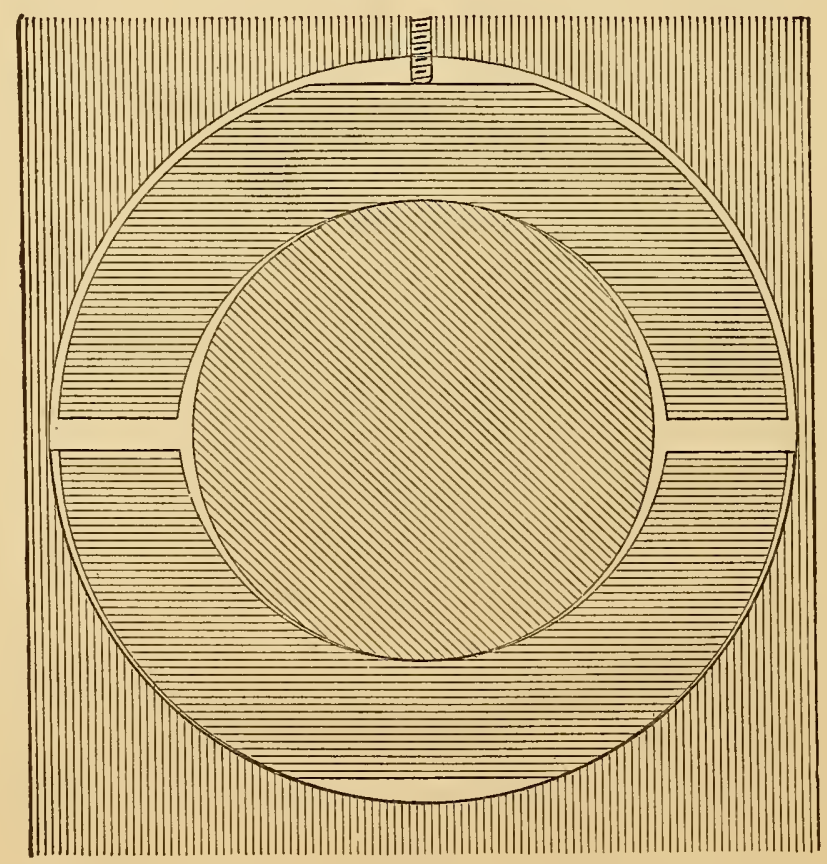

Fig. 17. - Showing the Contact of a Round Rod held in a Round Hole by means of Bushings. 
of the magnetic circuit whose reluctance is to be small seems entirely unwarranted.

Fig. I 7 shows the form of contact in one quite widely used commercial permeameter. As shown here, one bushing is entirely out of contact with the body of the yoke, and the other bushing has a very limited surface of contact. Between the specimen and the bushing, likewise, the contact is quite limited. Such unnecessary increase in the number of joints in the cirtical part of the magnetic circuit may be avoided by using a specimen of rectangular section. Fig. I 8 shows a yoke designed for specimens with par-

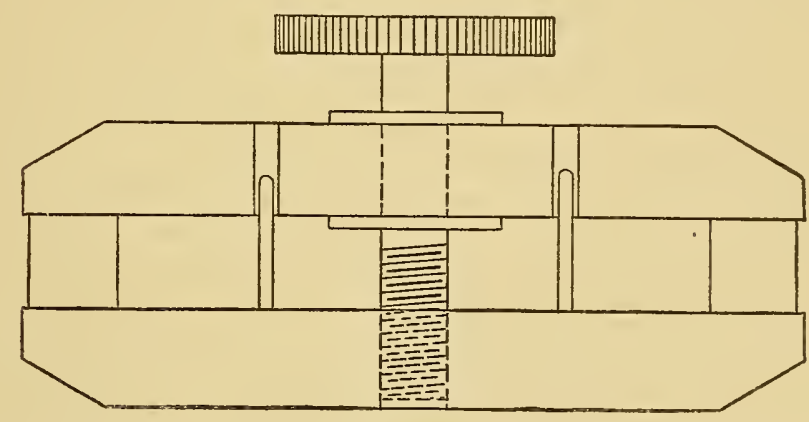

Fig. 18.- Showing the Perfect Contact of a Rectangular Specimen held between Plane Faces of a Parallel-jawed Yoke.

allel sides. Here a good contact is made regardless of the size of the specimen. The joint between the two parts of the yoke does not increase the magnetic reluctance because it is parallel to the direction of flux. The edges have been chamfered off in order to eliminate, as far as possible, disturbing pole effects. The single screw is an advantage over two screws, as it reduces the time required to insert and clamp the specimen. The two pins in one half of the yoke working in holes in the other half keep the two halves of the yoke in alignment. The shoulder soldered to the under side of the screw holds the jaws of the yoke apart for the reception of the test rod.

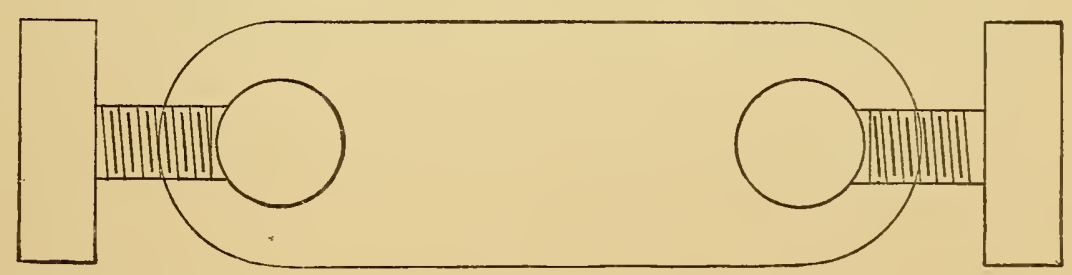

Fig 19.-Showing the Form 'of Yoke used with Round Specimens. The Thumb Screws are of Brass. 
If round specimens must be used Fig. I 9 shows a very satisfactory form. It is made of round iron, and the ends are turned down to hemispherical surfaces. The thumbscrews should be of brass.

It is possible, however, to use the same rod in a piece of apparatus which requires a circular section, and in another which requires a section of parallel opposite surfaces. A circularsectioned rod may have two parallel plane surfaces machined on it. These plane surfaces would form the contact surfaces in apparatus of one kind, and the curved surfaces would give practically as good contact in round holes as the whole cylinder.

In Fig. I4 the double hatching shows a part of the rod that is to be removed. It is not necessary to machine the whole length of the cylindrical rod. Those portions of the ends which form the contacts are sufficient.

Transformer iron is tested in the form of strips. The shearing of the metal modifies the properties of the iron, and results obtained with narrow strips are misleading. Extermely wide test pieces would be free from any appreciable influence of the shears, but would present other practical difficulties. Strips $5 \mathrm{~cm}$ wide

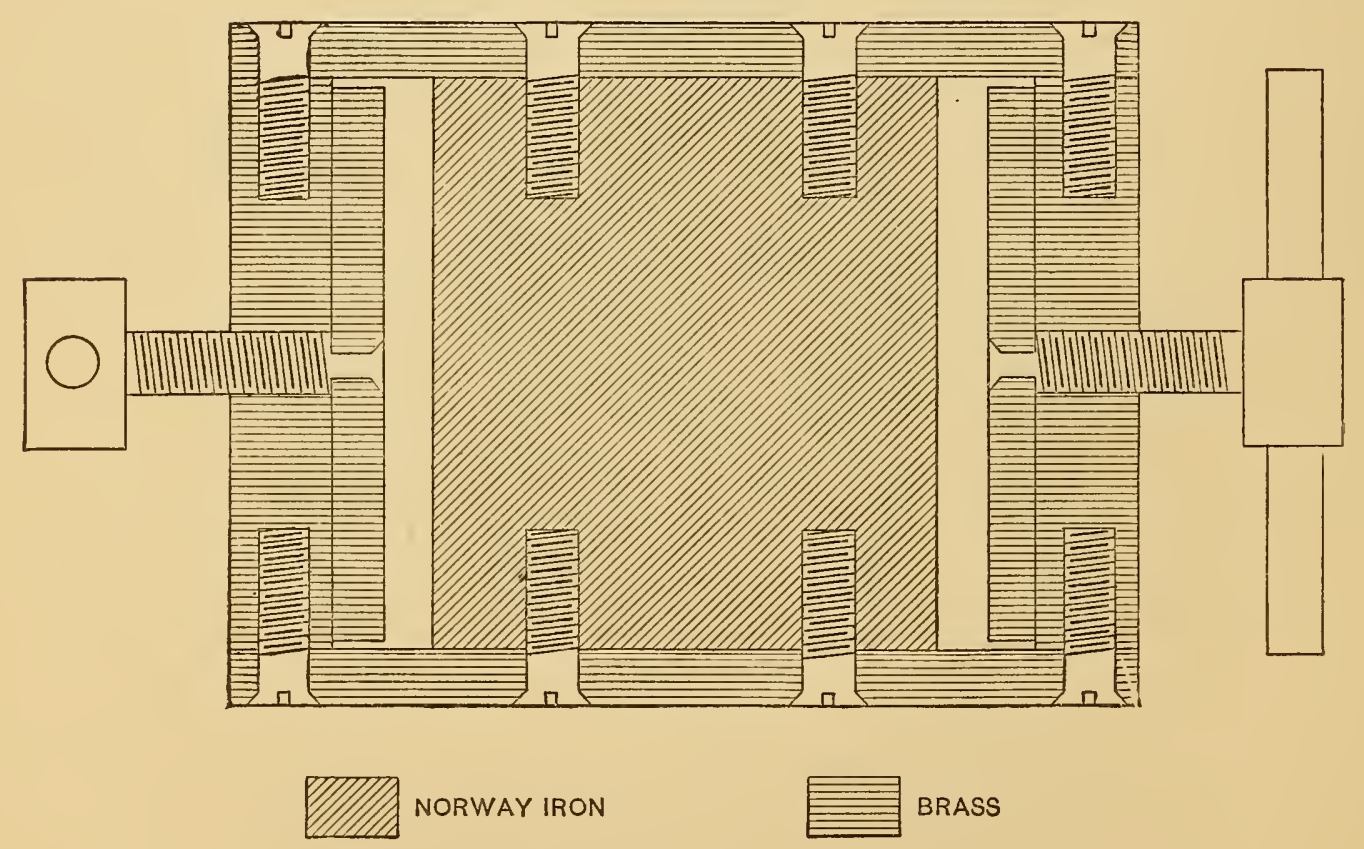

Fig. 20.-Showing the Form of Yoke used with Strips of Sheet Metal. 
can be readily handled, and will give a fair approximation to the magnetic properties of the unsheared metal and are recommended as a standard width of test strip.

A convenient form of yoke for such strips is shown in Fig. 20. Here the outside portions of the yoke which form the clamp are made of brass. If made of iron it would produce a greater disturbance of the leakage and probably not improve the magnetic joint appreciably.

\section{UNIFORMITY OF SPECIMEN.}

There may be irregularities in the flux distribution other than those caused by the yokes and joints. Fig. 2 I shows the flux distribution of a rod under different conditions of the complete magnetic circuit. In the curves of this figure the points of maximum induction have been shifted up or down along the vertical axis until the same point represents the induction at one end of the rod under all conditions. The scale unit is the same for all curves. The data for these curves were obtained by noting the deflection, on reversal of the magnetizing current, caused by the differential electromotive force in two test coils, one near the end of the bar and the other movable so that it occupied successively all points along the rod.

Instead of a single point of maxinum induction, there are two such maxima separated by a well-defined minimum. The minimum is most marked under conditions of the lowest reluctance in the yokes and joints. When the bar is reversed (curve $V$ ) while the yokes and coils remain unchanged, the section of minimum flux changes with the rod, so that this irregularity in flux is due to the rod itself and not to magnetizing solenoid or the yokes. An examination of the surface of this test specimen showed that an identifying number had been stamped very lightly upon the rod at the place where the minimum of flux appeared. Thus it would seem that the stamping had produced a hardening of the. metal at this point, and a consequent lowering of the permeability. It suggests that identifying marks should not be stamped on those portions of the test rod which are to be assumed homogeneous in the test. 


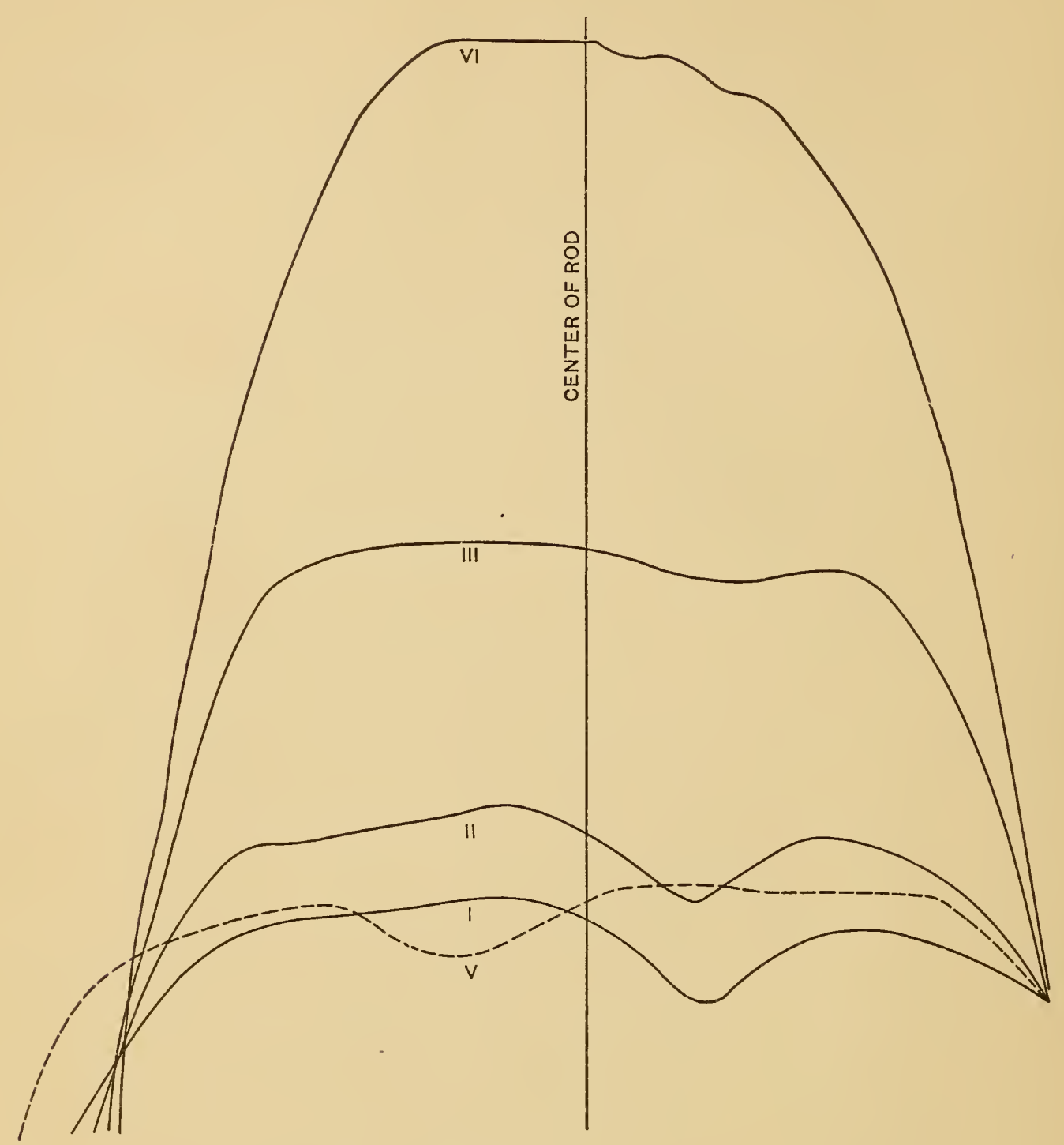

Fig. 21. - Showing Irregularities in the Flux due to Irregularities in the Test Bar itself in a Magnetic Circuit consisting of Two Rods and Two Yokes excited by a Constant Magnetomotive Force.

Curve I with yokes close to solenoids.

Curve II with yokes $1.5 \mathrm{~cm}$ from ends of solenoids.

Curve III with yokes removed.

Curve IV with test bar alone (yokes and auxiliary bar removed).

Curve V same as 1, but with test bar reversed. 
Fig. 22 brings out the same main points in a different way. A test rod was placed in a double bar and yoke magnetic circuit, and the flux along its length measured. Then the yokes and solenoid were moved along $5 \mathrm{~cm}$, relatively, to the rod, reclamped, and the flux distribution again measured. A second displacement of $5 \mathrm{~cm}$ was made, and another measurement taken. In the upper curve in the middle region of the bar, which here is surrounded by the middle of the magnetizing coils, are clearly

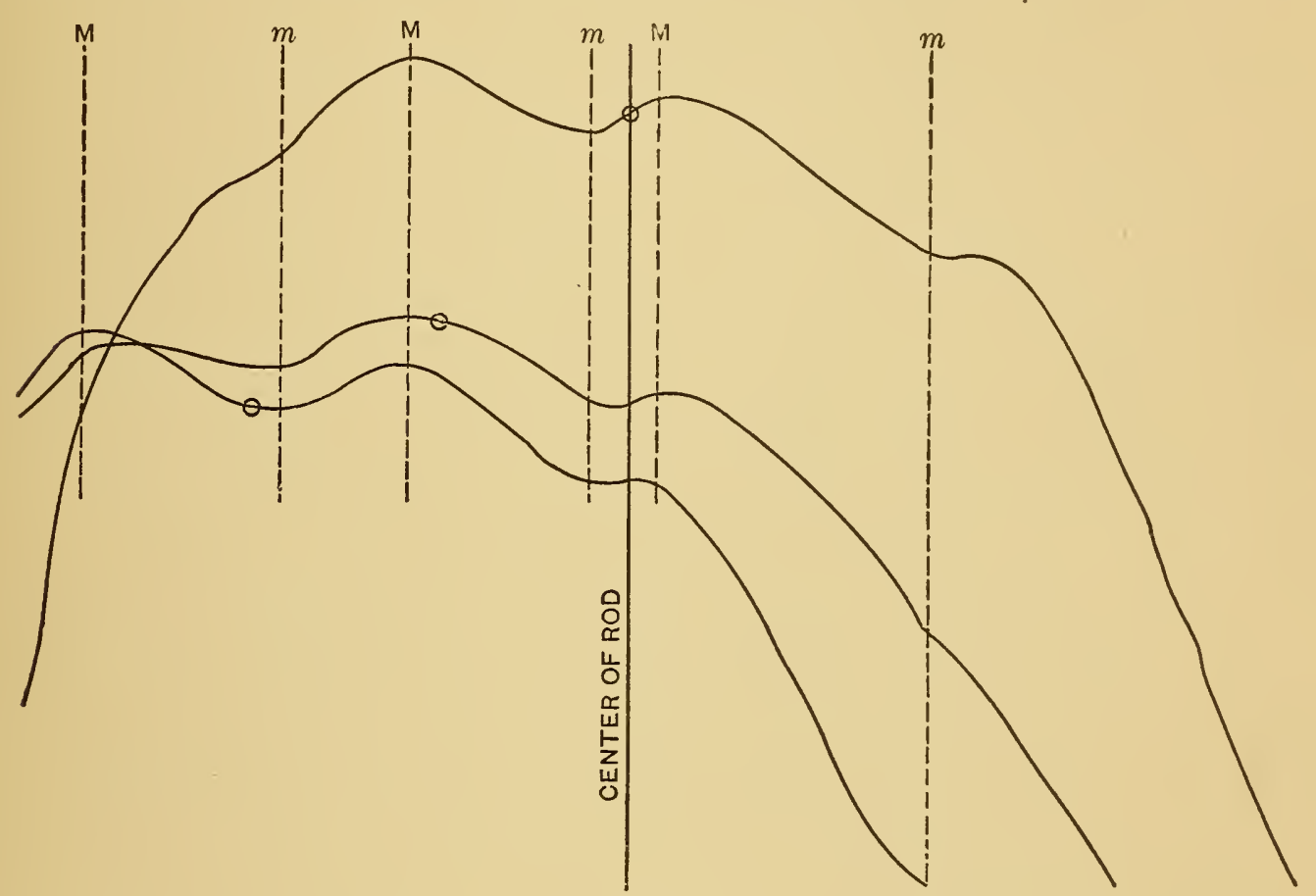

Fig. 22.-Showing the Flux Distribution for a Portion of a Rod $65 \mathrm{~cm}$ long, used in a Double Yoke Apparatus with 42-cm Coils for Three Different Relative Positions of Rods and Yoke.

The small circles show position of the midpoint of magnetizing coil.

seen two flux maxima and three flux minima. In the lower curves, where the magnetizing coils have been shifted, relatively, to the left so as to bring these points nearer the yokes, these same maxima and minima persist, but are less marked as they are approached by the yokes. Here again the general conclusion is that the rod produced some flux irregularity independent of that due to yokes or coils. A careful examination, visually, of this specimen failed to reveal any peculiarities at the points of maxima and minima.

Finally a rod was found which showed no marked irregularities. 2I92-No. $\mathrm{I}-\mathrm{O} 9-5$ 
This rod was stamped in three places with steel numbering dies, and then reexplored for variations in its flux distribution. Fig. 23 shows the flux distribution before and after stamping. The irregularities in flux distribution are here very marked.

From what has just been shown regarding flux distributions, it is quite evident that specimens which are to be accurately measured and preserved as standards must be handled carefully. It need not be surprising that a bar has slightly different magnetic properties after it has been dropped on the floor. Identifying numbers should not be stamped on the middle portion of the

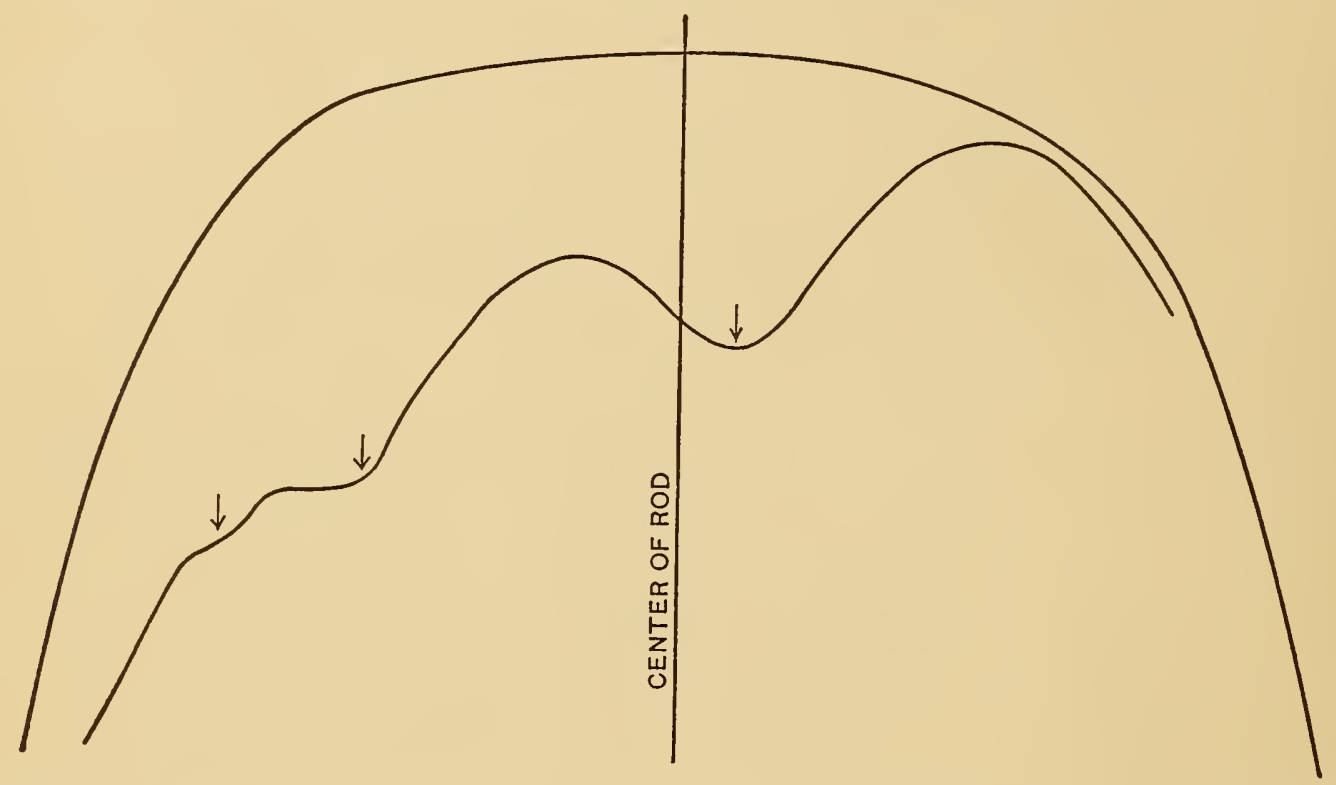

Fig. 23.-Showing Irregularities in Distribution of Flux in a Rod which has been Rendered Nonhomogeneous by Stamping Numbers at the Points Indicated by Arrows.

The upper curve shows distribution in homogeneous rod before stamping.

standard. It is objectionable to stamp them on the surfaces which are to form the magnetic joint with the yoke. The end of the rod seems the best place for the number. Placed here it has the further advantage of being visible when clamped in the yokes.

\section{DISTRIBUTION OF THE TEST COILS.}

Even with care in the selection and preservation of standard rods, irregularities are sure to occur. Consequently, the data obtained must be of necessity of the nature of mean values. To secure a reliable mean value, the test coils should be distributed over an appreciable length of rod. 
It is found in practice that if uniformity of induction is secured over the middle section of the test and auxiliary rods, and over a section near the end of the test rod in the manner indicated, the small irregularity of flux that may remain in the yoke may be neglected. It is well, however, to place what we have called the end test coils far enough away from the yoke to avoid the local irregularities in the immediate vicinity of the joint.

A very satisfactory distribution of the three test coils is as follows: The main test coil is wound closely over the middle quarter of the test specimen. On each side of this, midway between it and the yokes, are wound the two halves of a second coil. The third coil, similar to the first, is wound over the auxiliary rod. The three magnetizing coils, one over the test rod, a similar one over the auxiliary rod, and the third distributed in four parts over the four ends of the rods, are adjusted until the fluxes, linked by the three test coils, are equal. These test coils may conveniently be wound on thin cores made of paper, tracing cloth, or slotted metal. They should fit as closely as possible so as to have only a small correction for the flux that is within the test coil, but outside the iron. They may, of course, be wound on the same cores as the main magnetizing coils either inside or outside of the solenoid, but this disposition necessarily gives a larger correction for the flux linked by the coil but not passing through the iron, and is not to be recommended.

\section{DIRECT READING METHOD OF MEASURING THE MAGNETIZING FORCE.}

Having thus secured uniformity of flux in the test specimen, we may treat our magnetic circuit, as far as the middle section of the test bar is concerned, as though we had a uniform bar of infinite length surrounded by a uniform solenoid of equal length. In such a system the magnetizing force and magnetic induction may be readily calculated.

This assumption of equivalence to an infinite solenoid introduces no appreciable error. In Fig. 24 the magnetizing force at the center of the magnetizing solenoid is due to the main solenoid, the compensating turns, and whatever free poles may be developed in the iron itself. When the compensation is adjusted for uniformity of flux, the last force, of course, reduces to zero. 
That due to the coils is approximately equivalent to an indefinite uniformly wound solenoid. To determine how closely this approximation holds, let us consider the corrections which must be applied to the actual field at the center of the finite solenoid to produce the ideal field of the infinite solenoid.

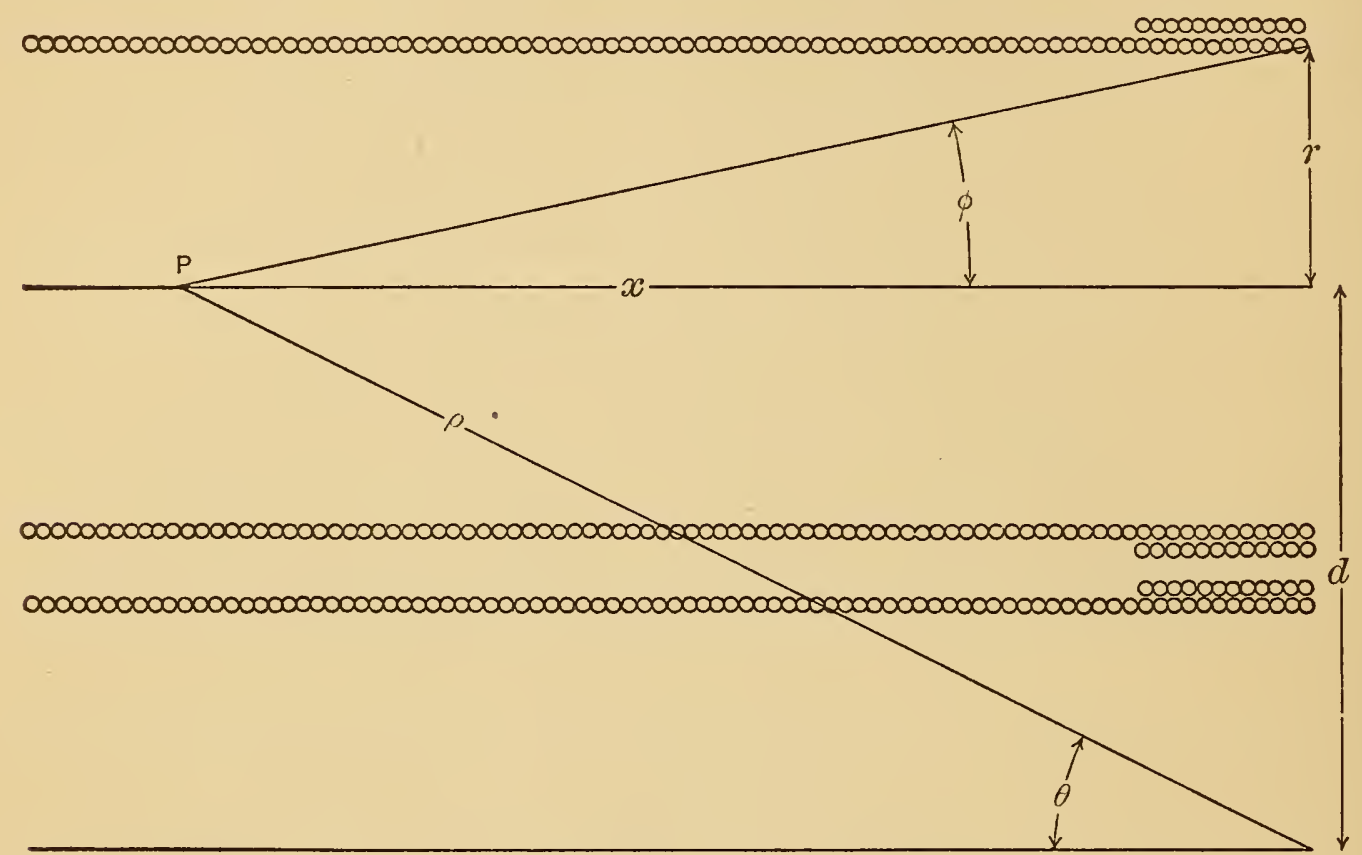

00000000000000000000000000000000000000000000000000000000000000000000000000000000000000000000

Fig. 24.

It may be shown that the correction due to one end of the solenoid $A$ is $-\frac{\mathrm{I}}{2}(\mathrm{r}-\cos \phi) H_{0}$ where $H_{0}$ is the field due the infinite solenoid. Expanding this (see Fig. 24).

$$
\begin{aligned}
-\frac{\mathrm{I}}{2}(\mathrm{I}-\cos \phi) H_{0} & =-\frac{H_{0}}{2}\left(\mathrm{I}-\frac{x}{\sqrt{x^{2}+r^{2}}}\right) \\
& =-\frac{H_{0}}{2}\left(\mathrm{I}-\left[\mathrm{I}-\frac{\mathrm{I}}{2}\left(\frac{r}{x}\right)^{2}+\frac{3}{8}\left(\frac{r}{x}\right)^{4}-\frac{5}{\mathrm{I} 6}\left(\frac{r}{x}\right)^{6}+\cdots-\cdots\right]\right) \\
& =-\frac{H_{0}}{2}\left[\frac{\mathrm{I}}{2}\left(\frac{r}{x}\right)^{2}-\frac{3}{8}\left(\frac{r}{x}\right)^{4}+\frac{5}{\mathrm{I} 6}\left(\frac{r}{x}\right)^{6}-\cdots\right]
\end{aligned}
$$


To get the force along the same axis due to one end of the other solenoid, we replace $x$ by $\rho$ and multiply each term by the appropriate zonal harmonic of $\cos \theta$. As the second solenoid develops a field opposite to $H_{0}$ we have for the correction due to one end of solenoid $B$

$$
+\frac{H_{0}}{2}\left[\frac{\mathrm{I}}{2}\left(\frac{r}{\rho}\right)^{2} P_{1}-\frac{3}{8}\left(\frac{r}{\rho}\right)^{4} P_{3}+\frac{5}{16}\left(\frac{r}{\rho}\right)^{6} P_{5}-\cdots\right]
$$

For the correction at the center of the solenoid $A$, due to one of its ends for a particular set of coils in which

Eq. (I) gives

$$
\begin{aligned}
& r=1.6 \mathrm{~cm} \\
& x=15 \mathrm{~cm} \\
& d=6 \mathrm{~cm} \\
& \theta=22^{\circ}
\end{aligned}
$$

$$
\begin{aligned}
& -\frac{H_{0}}{2}\left[\frac{\mathrm{I}}{2}\left(\frac{\mathrm{I} .6}{\mathrm{I} 5}\right)^{2}-\frac{3}{8}\left(\frac{\mathrm{I} .6}{\mathrm{I} 5}\right)^{4}+\cdots \cdot-\cdot\right] \\
= & -\frac{H_{0}}{2}[0.00568-0.00005+\cdots \cdots-\cdot] \\
= & -0.0028 H_{0}
\end{aligned}
$$

Eq. (2) gives

$$
\begin{aligned}
& +\frac{H_{0}}{2}\left[\frac{1}{2}\left(\frac{1.6^{2}}{15^{2}+6^{2}}\right) 0.9272-\frac{3}{8} \frac{1.6^{4}}{\left(15^{2}+6^{2}\right)^{2}} 0.6019+\cdots-\cdots\right] \\
& =+\frac{H_{0}}{2}[0.00454-0.0002+\cdots \cdots-\cdots] \\
& =+0.0023 H_{0}
\end{aligned}
$$

The total correction due to the four ends is therefore

$$
2(-0.0028+0.0023) H_{0}=-0.0010 H_{0}
$$

To determine the force at the center of a main solenoid due to the compensating coils, substitute the appropriate constants for each end of the compensating coil. In the particular case considered- 


$$
\begin{aligned}
& \text { For inner end. } \\
& r=2.5 \mathrm{~cm} \\
& x=\mathrm{I} 3 \mathrm{~cm} \\
& d=6 \mathrm{~cm} \\
& \theta=\tan ^{-1} \frac{6}{\mathrm{I} 5}=22^{\circ} \quad \theta=\tan ^{-1} \frac{6}{\mathrm{I} 3}=25^{\circ} \\
& \text { For other end. } \\
& r=2.5 \mathrm{~cm} \\
& x=\mathrm{I} 5 \mathrm{~cm} \\
& d=6 \mathrm{~cm} \\
& \frac{\mathrm{I}}{2} \Delta H=-\frac{H_{\mathrm{o}}}{2}\left[\frac{\mathrm{I}}{2}\left(\frac{2.5}{\mathrm{I} 6}\right)^{2}-\frac{3}{8}\left(\frac{2.5}{\mathrm{I} 5}\right)^{4}+\cdots \cdot \cdot\right] \\
& +\frac{H_{\mathrm{o}}}{2}\left[\frac{\mathrm{I}}{2}-\left(\frac{2.5}{\mathrm{I} 3}\right)^{2}-\frac{3}{8}\left(\frac{2.5}{\mathrm{I} 3}\right)^{4}+\cdots \cdot . . .\right]
\end{aligned}
$$

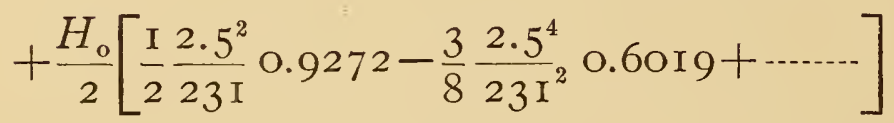

$$
\begin{aligned}
& -\frac{H_{0}}{2}\left[\frac{\mathrm{I}}{2} \frac{2.5^{2}}{\mathrm{I} 75} 0.9063-\frac{3}{8} \frac{2.5^{2}}{\mathrm{I} 75^{2}} \mathrm{O} 0.5016+\cdots \cdots\right] \\
& =\frac{H_{0}}{2}\{-(0.0139-0.0003)+(0.0185-0.0005) \\
& +(0.0125-0.0002)-(0.0161-0.0002)\} \\
& =+0.0004 H_{0}
\end{aligned}
$$

where $1 / 2 \Delta H$ is the force at the center of one of the solenoids due to the compensating coils at one end for the same current turns per cm. as the main solenoids. As there are two such sets of compensating coils, and experiment has shown that at times they carry twice the current turns per unit length of the main solenoids, we may expect a maximum field at the center of main solenoids, due to these four compensating coils of +o.00I6 $\mathrm{H}_{0}$. Combining this correction with that due to the ends of the main solenoids we find a maximum correction of less than o. I per cent.

For a shorter set of coils $20 \mathrm{~cm}$ long, but with the other dimensions the same, we find for the corrections due to the ends of the magnetizing solenoids and to the compensating turns $-0.005 \mathrm{H}_{0}$ and $+0.007 H_{o}$, respectively, so that these short coils may be used in work of a I per cent tolerance.

The remainder of this paper will be devoted to an account of certain modifications of the usual methods of measurement 
which have been found convenient, especially where the apparatus may remain permanently set up.

Magnetizing coils, for use in laboratory work, are very frequently wound with no reference to ease of calculation of the magnetizing forces. The magnetizing force is given by the formula $H=0.4 \pi n I$ where $n$ the number of turns per $\mathrm{cm}$. is fixed, and $I$ is variable. With haphazard values of $n$, the factor of proportionality between field strength and current will be an awkward number to handle. The computations are much simplified if the number of turns per centimeter is so chosen that this factor of proportionality is a power of $\mathrm{IO}$. This is accomplished by making $n=7.958$ turns per $\mathrm{cm}$, whence $H=\mathrm{IO} I$. For $n=79.58, H=\mathrm{IOO} I$. Both these values of $n$ are used in various commercial permeameters, and while very convenient, they offer considerable difficulty to the mechanician.

Another method of avoiding the labor of a long computation is to vary the current by a number of fixed steps in the regulating rheostat. If the total emf. impressed on the coil and regulating rheostats is constant, each value of the regulating resistance corresponds to a fixed value of magnetizing force. If, further, the divisions of the rheostat are adjusted so that integral values of $H$ are given, we have a very desirable arrangement. Some means of maintaining constant potential difference must be employed. This and the lack of flexibility more than counterbalance the advantages of this method of regulation.

We may vary the magnetizing force by maintaining the current constant and varying the number of active turns. This method has the same advantages and disadvantages as the preceding and has the further objection that irregularities in the magnetizing force occur unless special precautions are used in winding. This method of varying the magnetizing force is used in some commercial apparatus.

In any given case we may determine the strength of the magnetizing current by measuring the fall of potential over a standard resistance of suitable value. The difference of potential can be measured to a high degree of precision by means of the potentiometer and standard cell. This method has great flexibility and with the following modifications offers many advantages. 
The magnetizing force is given in terms of the fall of potential, $E$, over a given resistance, $R$, by the formula: $H=\frac{0.4 \pi}{R} n E$. If several magnetizing coils are to be used, from time to time, we can not expect $n$ to be the same for all coils. Even for the same nominal value of $n$ there may be variations of more than one-half per cent from this value unless the coil be wound with greater care than is ordinarily given to such work. We must, therefore, consider $n$ as a variable as well as $E$. If $R=0.01257 n$ then $H=100 E$. By arranging a slide wire in parallel with a resistance coil of such value that the combined resistance is $1.257 \mathrm{ohms}$, the drop of potential over $\frac{n}{\mathrm{IOO}}$ of the slide wire will be $E$. The reading of the potentiometer thus gives $H$ by simply shifting the decimal point. This arrangement is shown in Fig. 25, where $R_{0}$ is a slide-

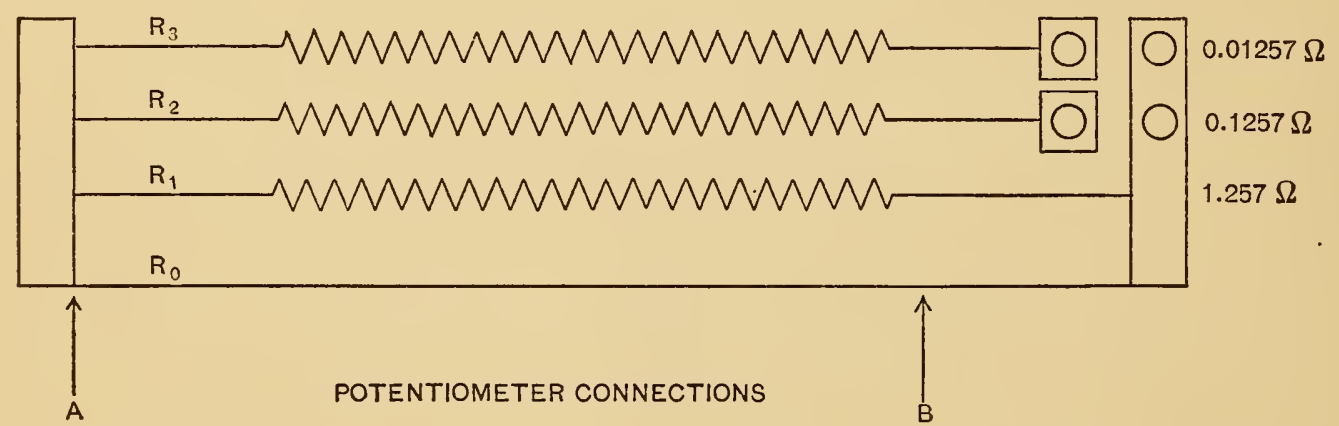

Fig. 25.-Showing Diagrammatically a Resistance Suitable for use with Potentiometer in Measuring the Magnetizing Force.

$\mathrm{R}_{0}$, a slide wire divided into 1,000 parts.

$R_{1}, R_{2}$, and $R_{3}$ resistances of such value that the total parallel resistances over $R_{0}$ and $R_{1}$ over $R_{0}, R_{1}$, and $R_{2}$, and over $R_{0}, R_{1}$, and $R_{3}$ are $1.257,0.1257$, and 0.01257 ohms, respectively.

wire bridge wound on a drum and divided into $\mathrm{I}, \mathrm{OOO}$ numbered parts, each subdivided into halves. The resistance, $R$, is adjusted to such a value that the total parallel resistance over $R_{0}$ and $R_{1}$ is r.257 ohms. If, now, the potentiometer is connected to the fixed contact $A$, and the sliding contact $B$, and this sliding contact is set at a point $=\frac{\mathrm{rO} n}{\mathrm{IOOO}}$, we shall measure $E$. This setting needs to be made only once for each coil. The magnetizing force is now $H=\mathrm{IOO} E$, where $E$ is the potentiometer reading. 
It may happen that a magnetizing current is required which would give a potential drop beyond the range of the potentiometer. In this case a second resistance, $R_{2}$, is added in parallel, so that the total parallel resistance becomes 0.1257 ohm. For extreme cases a third parallel resistance may be added to reduce the total resistance to o.or 257. This last resistance would be required only for magnetizing coils of few turns per centimeter, where the large current required would produce excessive heating of the higher resistance.

In case all work is to be done with one set of magnetizing coils or with several sets all wound with the same number of turns per centimeter, it may be desirable to have a fixed resistance of the proper value to make the potentiometer direct reading.

\section{THE MAGNETIZING COILS.}

If the solenoid is wound in a single layer, it offers several advantages. It is more easily wound and its uniformity more readily determined. If we wish $H=\mathrm{IO} I$, we must have 7.958 turns per centimeter. No. I 8 wire gives approximately this value. ${ }^{4}$ Such a coil will carry 5.4I amperes for a $30^{\circ} \mathrm{C}$. rise in temperature; that is, with a single layer values of $H$ up to 54 may be used continuously. For short intervals, long enough to take a measurement, double this value may be used. A solenoid of ten layers of the same wire will stand continuously I.7I amperes, which means $H=I 7 I$. Such a coil may be used for magnetizing forces up to 350 for short periods only.

The coils used in the precision magnetic work of the bureau have been made with great care. No. I 8 double-cotton-covered copper wire was used, the first layer being wound on a screw thread cut by a lathe. Succeeding layers wound in the same direction, each wire being guided by the depression between adjacent wires in the previous layer and the ends of each layer being separately secured. There are ten layers in all. As each layer was finished it was examined very carefully, and in no case had any deviation from 8 turns per centimeter been observed. When the winding was finished, the ten layers were connected in series. We may safely assume that the field at the center of this coil can be calculated to within

${ }^{4}$ Foster's Electrical Engineer's Pocket Book, p. 89, ed. 1902. 
o. I per cent. These coils give at the center a value of magnetizing force $H=100,53 I$. As this current is measured by the fall of potential $E$ over a resistance $R=\mathrm{I} .0053 \mathrm{ohms}$, we have the working formula

$$
H=\mathrm{IOO} E
$$

\section{MEASUREMENT OF THE INDUCTION.}

Formula for the induction.-When measured by a ballistic galvanometer, calibrated by a mutual inductance, the formula used to calculate the magnetic induction is

where

$$
B=\left(\frac{M I_{10} 0^{8}}{N_{2} A d_{0}}\right) d-\frac{a-A}{A} H
$$

$B=$ Flux per square centimeter.

$M=$ Mutual inductance in henrys.

$I=$ Current in amperes in the mutual inductance.

$N_{2}=$ Number of turns in test coil.

$d_{0}=$ Galvanometer deflection due to mutual inductance.

$d=$ Galvanometer deflection due to test coil.

$A=$ Cross section of iron.

$a=$ Cross section of test coil.

$H=$ Magnetizing force.

The Galvanometer.-The galvanometer must be of sufficiently long period that the full effect of the induced electromotive force of the test coils is transmitted to the moving system before it has moved very far from zero position. For rapidity of working, it is desirable that the moving system be aperiodic and the zero constant. The Thomson galvanometer is open to the objections that the zero is shifting continually, and often erratically, and that its sensibility varies with every change in the earth's horizontal intensity, and finally that the damping is tedious. The D'Arsonval is a more satisfactory instrument, as it is open to none of the above objections. It does have a lower sensibility, but this is sufficiently high for most ballistic purposes. The ease with which its sensibility is controlled and critical damping secured makes it a very desirable instrument in magnetic testing. For some work 
an instrument of the flux-meter type, in which the suspension exerts no control, is desirable, but at the pressent time mechanical imperfections and difficulties leave much to be desired in these instruments.

Calibration of the Galvanometer.- Several methods of calibrating a ballistic galvanometer have been given, but I have obtained the best results by using a mutual inductance. This has the very great advantage that its secondary coil may remain a part of the galvanometer circuit, not only during calibration but also during the regular ballistic measurements. Thus the galvanometer is calibrated under normal working conditions. Furthermore, a standard of mutual inductance is inexpensive, simple in construction and use, permanent in its value, and if the primary is a long solenoid its value may be readily calculated from its dimensions.

Simplicity in calibration corrections may frequently be obtained by using only the lower part of the scale. In some of the earlier work of the present investigation the galvanometer sensibility was reduced until $\mathrm{I} \mathrm{cm}$ deflection corresponded to Iooo in $B$. This gave a maximum deflection in the average practical test of I $7 \mathrm{~cm}$ corresponding to $B=\mathrm{I} 7000$. The least reading, O.I $\mathrm{mm}$., corresponds to ro gausses. With this contracted range the calibration corrections were always less than 0.5 per cent and were usually negligible, thus permitting a great saving of time.

Unfortunately a galvanometer does not retain its calibration. The actual value of the induction corresponding to a particular deflection as well as the form of the calibration curve vary within small limits. Temperature changes may modify the strength of the magnet, the torsion coefficient of the suspension, the length of the suspension, the cross section of the coil, as well as the total resistance of the circuit. These variations are of sufficient importance to warrant a determination of the ballistic constant at the beginning and at the end of each run. Obviously, any mechanical adjustment of the suspension or of the galvanometer will produce still greater variations.

Another source of trouble in deflections taken with a galvanometer having an iron core is the effect of eddy currents and hysteresis. If the secondary of a mutual inductance is connected 
in series with a galvanometer, it is found that the deflection due to the reversal of a given primary current is less for very rapid reversal than for a somewhat slower one. To prove that this difference is due to the iron in the galvanometer core, the galvanometer circuit was made to include a solenoid into which a bar of iron could be placed. In this case the galvanometer showed a smaller deflection when the iron was within the solenoid than when it was not, even when the rate of reversal and other details of the experiment were unaltered.

In one case the difference with and without iron, in the solenoid, was 0.25 per cent. The explanation of the difference in deflection in these two cases is probably to be found in the increase of effective resistance of the galvanometer circuit due to hysteresis and eddy currents. These latter will each be greater for the greater secondary current flowing, and since the integral of the electromotive force due to a given change in the primary current of a mutual inductance is constant, the instantaneous values of the induced electromotive force and, consequently, the current will be greater the shorter the time of integration, or in this case the time of reversal. The hysteresis and eddy currents thus reduce the galvanometer deflection. However, if the same methods of manipulation are followed in the determination of the constant and in the test, no serious error will result.

The Mutual Inductance.-The factors which enter into the value of $B$ can not all be determined to the same precision. The mutual inductance may easily be determined to I part in Ioooo. As an illustration, one pair of coils gave for the value of the mutual inductance, as determined on different days, values of I 5.2480 and I 5.2486 millihenrys, respectively, a difference of I in 25000. The mutual inductance of these coils has not changed by a measurable amount since they were first made and measured three years ago. In using the mutual inductance it is necessary that all leads to both primary and secondary be twisted in pairs in order that each circuit may neither envelop nor develop any accidental magnetic flux. This precaution is particularly important close to the mutual inductance. This mutual inductance must be placed far enough away from the magnetic circuit under test, so that no stray field may act upon its secondary and 
thus cause an extra electromotive force to be generated in the galvanometer circuit at the instant the ballistic measurement is made. Errors may be introduced into the ballistic readings if from any cause there is a sudden change in the magnetic field at the place where the mutual inductance is located. This source of trouble was forced upon my attention while working with a standard of mutual inductance approximately 20 centimeters in diameter and having several hundred turns in the secondary. With this coil in circuit with the galvanometer every movement of an elevator some 30 meters distant was indicated by the galvanometer. This disturbance was probably due to the change in vertical length of the steel supporting cable as it coiled and uncoiled upon the cable drum. The lower end of the cable is undoubtedly magnetized as a north-seeking pole by the terrestial field, and as the elevator moves in its shaft the flux from this moving magnet sweeps through the surrounding space. The trouble disappeared when the plane of the coil was placed vertical instead of horizontal. This suggests that any disturbance due to a varying field may be eliminated by placing the coil so that its turns do not encircle the varying flux. This position can readily be ascertained by trial.

The Current.-The current through the primary of the mutual inductance may be determined with any precision required. Throughout the present work all current measurements were made by measuring the fall of potential over a standard resistance by means of a potentiometer.

Test Coils.-No appreciable error need appear in the value of $N_{2}$ if the leads are twisted. There may be loops in the leads and some stray flux may thus be caught. Increasing the number of test turns reduces any such effect. Care must be taken that there is no short circuiting of secondary turns.

The Cross Section of Specimen.-A, the cross section of the test specimen, may be an important source of error in the determination of the induction, especially in samples of thin transformer iron. As the specimen comes from the lathe, the milling machine, or the rolls and shears if in sheet form, it is not of uniform section but varies by as much as several hundredths of a millimeter. If this variation tapers from one end to the other the error is mostly self-compensating. Sheared metal furnishes the 
most objectionable irregularities and milled metal the least. The actual dimensions of the cross section can easily be measured to a hundredth of a millimeter. This would mean about I or 2 per cent for thin transformer iron and one-tenth as much for iron several millimeters thick. The difficulty does not end here, however, as the specimen is often grooved and pitted. The micrometer measures the maximum dimensions and hence may indicate a larger cross section and a correspondingly smaller induction is computed. The error due to this last irregularity is eliminated by getting the specific gravity, weight, and length, and from these calculating the cross section. In one set of specimens I cm wide by $0.035 \mathrm{~cm}$ thick and $5 \mathrm{I} \mathrm{cm}$ long the density method indicated a cross section 6 per cent less than that of direct micrometer measurement.

Air Flux.-The correction $\frac{a-A}{A} H$ is for the flux which passes through the test coil but outside the iron. This should be made small by winding the test coils close to the specimen, in the case of small specimens. If the specimen is of large cross section and nearly fills the magnetizing coil, and this is of a single layer, the test coil may be wound outside this primary coil. As an illustration of the magnitude of this correction, suppose the test coil I.5 $\mathrm{cm}$ in diameter and the specimen $0.5 \mathrm{~cm}$ in diameter. Then the correction is $8 H$ in $B$, or 8 in the permeability. For a sheet of transformer iron .03 by $\mathrm{I}$ in the same test coil, the correction is $58 H$ in $B$, or 58 in the value of $\mu$. An error of .or $\mathrm{cm}$ in the diameter of the test coil would introduce an error in $B$ of $0.8 \mathrm{H}$. This is equivalent to an error of I per cent at those parts of the induction curve where the permeability is 80 . Consequently it is inadvisable to have the test coil wound outside the primary when specimens of very small section are to be tested.

It is possible to simplify this correction by having a second magnetizing coil and test coil arranged similarly to the working coils but without any iron inserted. The same magnetizing current flows through each primary coil and the two secondaries are so connected that the induced emfs. oppose each other. In such an arrangement the galvanometer is not affected by any change in the magnetizing circuit until the iron rod is inserted in one coil 
and its deflections are due to the increase of flux due to the iron alone. To the value of $B$ thus determined must be added the magnetizing force, but as this correction term is always small and requires no calculation it is not at all troublesome. As these coils constitute a mutual inductance, any form of adjustable inductance might be used and the proper value determined by noting when the galvanometer showed no deflection without iron in the magnetizing coil.

\section{ZERO METHOD.}

In discussing the influence of errors in the galvanometer deflections and scale calibration, it was noted that these errors were minimized if the deflection due to the mutual inductance in getting the galvanometer constant was equal to the deflection produced by the change of induction in the iron. This suggests that it might be desirable to impress both these electromotive forces in opposition simultaneously on the galvanometer. The galvanometer would indicate the equality of the two integrated electromotive forces by a zero deflection. If this is done the induction, neglecting the correction term, becomes

$$
B=\frac{M I_{10} 0^{8}}{N_{2} A}
$$

As $N_{2}$ and $A$ are constant for any given test coil and specimen, this formula becomes

$$
B=M I \times \text { constant }
$$

If we use this equation and vary both $M$ and $I$ it is desirable to have the same current $I$ flow through the magnetizing coil and the primary of this balancing inductance. Since further

$$
H=I \times \text { constant }
$$

we get by dividing (3) by (4)

$$
\mu=\frac{B}{H}=M \times \text { constant }
$$

so that in this method the values of permeability are obtained very readily from $M$ after a single calculation. 
Instead of varying both $M$ and $I$ we may keep $I$ constant and vary $M$ alone. This offers some advantages when the same sized specimen is always used. In this case we have

$$
B=K M
$$

The inductance may be calibrated to read $B$ directly.

We may, however, keep $M$ constant and vary $I$. The formula (3) now becomes

$$
B=I \times \text { constant }
$$

If, further, $M$ is adjustable through a wide range it may be given such a value that the constant becomes a power of Io. In this case the induction is obtained directly from the current $I$ by merely shifting the decimal point.

The value of mutual inductance required is

$$
M=\frac{N_{2} A}{{ }_{10}^{8}} \times \text { constant }
$$

In the present work the constant chosen is $\mathrm{IO}^{4}$, so that $M=\frac{N_{2} A}{\mathrm{IO}^{4}}$ and $B=10000 I$. Since $N_{2}=$ I00, $M=0.0 \mathrm{I} A$.

As the current measurement is made by determining the fall of potential over a standard resistance, the above formula becomes $B=\mathrm{I} 000 \mathrm{O} \frac{E}{R}$, or for a resistance of $\mathrm{I}$ ohm $B=\mathrm{I} 0000 E$. Thus the flux density determination depends upon a potentiometer setting.

First among the several advantages of this manner of measuring the induction is the fact that it is a zero method and as such shares all the merits usually possessed by zero methods. It is independent of the galvanometer scale calibration and the galvanometer may be worked at its maximum sensibility, usually many fold that permitted by the deflection method. It permits one to set the mutual inductance current at a value corresponding to any chosen value of the induction, after which $H$ may be adjusted to correspond. This is of particular importance in the double yoke method, in which the two magnetizing forces used to produce the same induction in different lengths of specimen are compared. 


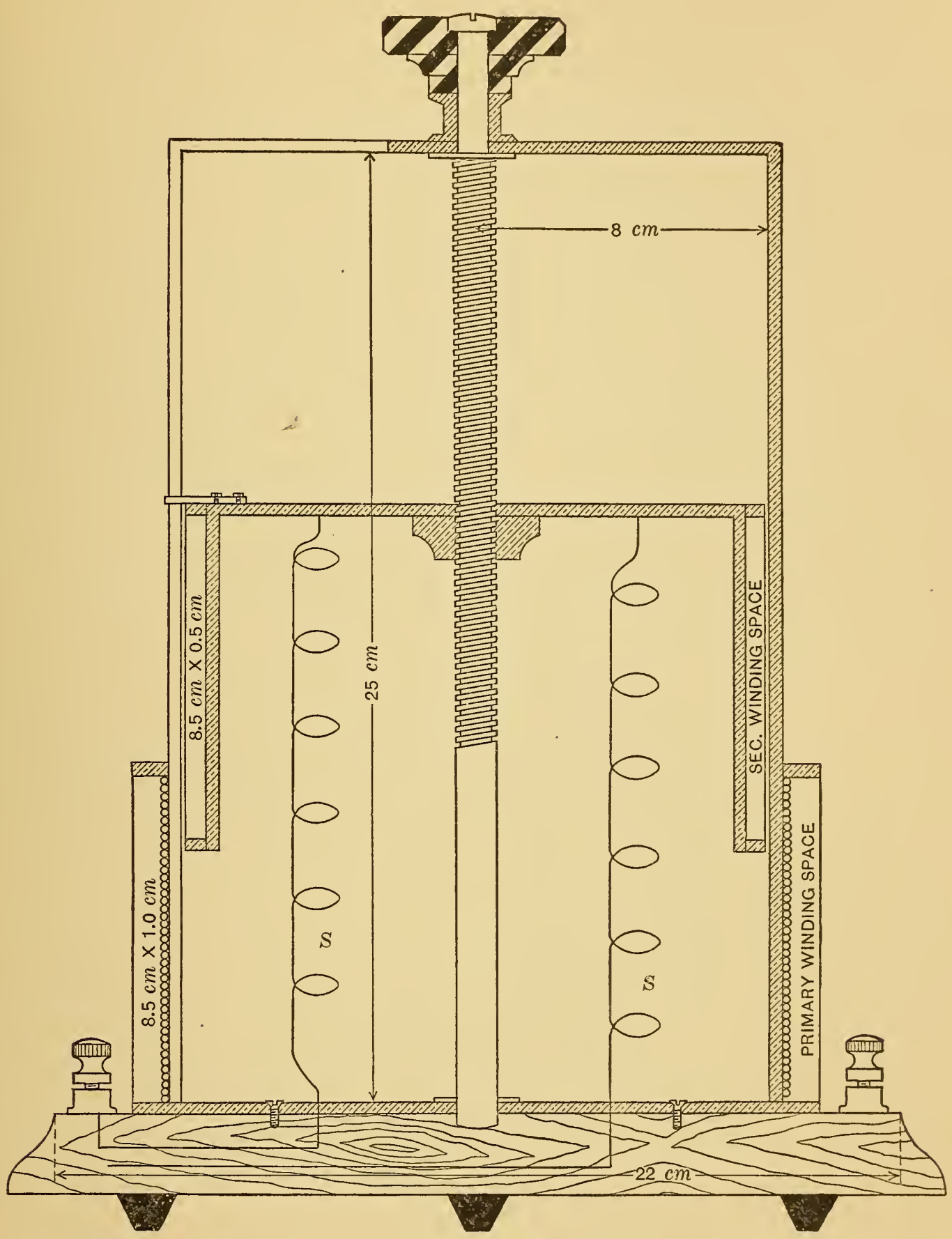

Fig. 26.-Sectional View of a Variable Mutual Inductance designed especially for use in Magnetic Testing.

$2 \mathrm{I} 92-$ No. $\mathrm{I}-\mathrm{O} 9-6$ 
By the deflection method it is necessary to plot the full curve for each length of rod and then measure the abscissa differences and finally lay off the resultant curve, which is the only one desired. By the zero method the balancing current is set to the required value of the induction for each length and the differences in magnetizing forces obtained directly from the data without the necessity of plotting the two auxiliary curves. In fact it is not necessary to plot any curve unless so desired.

It is also a decided advantage to know the corresponding values of $B$ and $H$ during the course of the experimental determination. Without this knowledge it is difficult to determine the proper spacing of the points of observation. By the deflection method it frequently happens that too few points are taken on the steep part of the curve and more than necessary in the upper ranges. In cyclic curves the points most frequently desired are the maximum induction, the residual induction, and the coercive force. The zero method enables one to set on these points directly, while in the deflection method the curve must be plotted before the coercive force is known.

\section{VARIABLE MUTUAL INDUCTANCE.}

Fig. 26 is a sectional view of a variable mutual inductance designed for use in magnetic testing. The primary is wound in six layers on one end of a brass cylinder slotted so as to reduce eddy currents. The terminals of this primary and tap wires from two intermediate points are brought out to binding posts mounted on the base. The secondary is wound on an inner cylinder which is drawn along the axis of the primary coil by a threaded shaft of phosphor-bronze. A small index moving in a slot cut in the outer cylinder prevents the inner spool from turning on its axis and also indicates its position. The terminals of the secondary are brought to binding posts in the base, through the spirals $S$.

The secondary coil has 1200 turns of No. 30 D. S. C. magnet wire, having a total resistance of $180 \mathrm{ohms}$ at $22^{\circ} \mathrm{C}$. The primary has three sections of No. 20 D. C. C. magnet wire, with the following constants: 


\begin{tabular}{c|c|c|c|c}
\hline $\begin{array}{c}\text { Designation of } \\
\text { Section }\end{array}$ & Number of Turns & $\begin{array}{c}\text { Maximum Mutual } \\
\text { Inductance }\end{array}$ & $\begin{array}{c}\text { Minimum Mutual } \\
\text { Inductance }\end{array}$ & Resistance \\
\cline { 2 - 4 } A & 6 & 0.89 & 0.13 & Ohms. \\
B & 55 & 7.8 & 0.8 & 0.17 \\
C & 543 & 75 & 7 & 1.07 \\
& & Mh. & 9.13 \\
\hline
\end{tabular}

Fig. 27 gives the calibration curves for the three primaries A, $\mathrm{B}$, and $\mathrm{C}$.

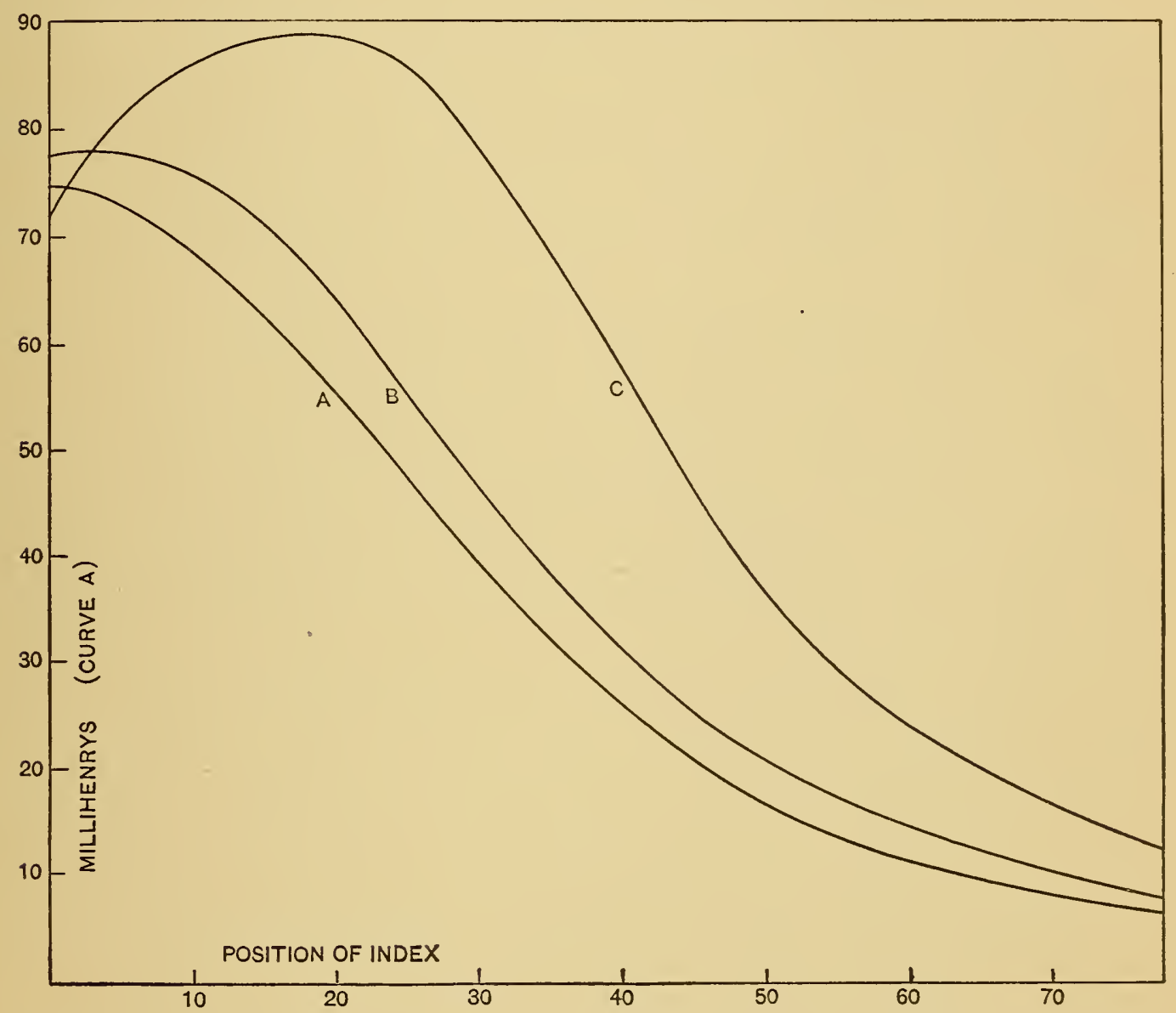

Fig. 27.-Showing Calibration Curves for the Variable Mutual Inductance shown in Fig. 26.

Curve $A$ is with the full 543 turns of primary winding.

Curve $B$ is with 55 turns of primary and the vertical scale is 0.1 that indicated on the left.

Curve $C$ is with 6 turns of primary and the vertical scale is 0.1 that indicated on the left.

From these curves we see that the ratio of the change in inductance to the displacement of the coil is a maximum when the coil is in its middle position. Even here, however, a change of o.I per 
cent in inductance corresponds to a rotation of a little over $7^{\circ}$ of the shaft by which the coil is moved. The peculiarity in the shape of the calibration curve for the 6 turns of primary is due to the fact that these turns are wound on the upper end of the primary so that they do not become most effective until the secondary has moved up far enough to be directly opposite these turns.

This variable mutual inductance can be set, with an accuracy of o. I per cent or better, to any value greater than o.I 3 millihenry and less than 75 millihenrys. As the current through the primary is to be related to the flux density in the iron by the formula $B=10000 I$, it is necessary that a current of 2 amperes produce no excessive heating if a flux density of 20000 is desired. A current of this value maintained for one hour produced a rise of $33^{\circ}$ in temperature as measured by the change in resistance of the primary. Consequently the current-carrying capacity is ample.

\section{FULL SET-UP AND OPERATIONS.}

After the test bars are inserted in the yokes, the circuit is thoroughly demagnetized by a cyclic magnetizing force of one period per second, which is gradually reduced from an initial intensity which carries the induction well beyond the point of maximum permeability to a final value somewhat lower than the lowest induction to be studied.

After demagnetization in this way, the lowest force to be used is applied and reversed many times until the iron is brought to a cyclic magnetic state, that is, until the reversal of the magnetizing force reverses the direction of magnetization without changing its magnitude. During this process of reversals the adjustment for uniformity of flux may be made, as the adjustment involves only small changes in the magnetizing force.

Having made the adjustments necessary to secure uniformity of flux and brought the iron to the cyclic state, the induction is measured by balancing the electromotive force induced on reversal of the magnetizing current against that induced in the secondary of a mutual inductance on reversal of its primary current, which is adjusted to give the required balance. In order to exclude any influence due to mechanical vibrations the entire magnetic circuit is mounted on a layer of felt. These lower values of the 
induction produced by small magnetizing forces are easily altered by incompleteness of demagnetization or failure to reduce to a cyclic state. As a check, therefore, after determining this first point on the induction curve, it is well to carry the iron again through the demagnetizing process, readjust the compensation, reduce to a cyclic state, and redetermine the point.

If the second determination does not yield the same result as the first on the initial induction, the full process should be repeated more carefully until concordant results are obtained.

Having thus secured satisfactory data for the lowest magnetizing current, it is not necessary to demagnetize again. The remaining points on the induction curve may be obtained by passing to the next higher magnetizing force, adjusting the main and compensating currents, repeating the reversals till a cyclic condition is reached, and so on till the required data are secured. The curve determined by these points is called the hormal induction curve.

Before determining the hysteresis loop the iron is demagnetized in the same manner as before the normal induction test, although the demagnetization may be omitted if the specimen has not been subjected since its last demagnetization to forces greater than the maximum used in the hysteresis cycle. The maximum magnetizing force is applied and the compensation adjusted as for a point on the normal induction curve. By suddenly inserting a separately adjusted rheostat in the main magnetizing circuit the magnetizing force can be reduced to any desired value. This, in general, means that new adjustments of the compensating currents must be made in order to maintain uniformity of flux at this second point. This is done most conveniently without disturbing the original adjustment by inserting in the proper circuits suitably adjusted series or parallel resistances, according to whether the particular current is to be lowered or raised. This adjustment is made by means of the same test coils and in a similar manner as the original adjustment. Before each change from the maximum magnetizing current to the lower value, however, the iron must be brought back to a cyclic state. A double reversal of the maximum current usually suffices for this. After this compensation is made the change in induction produced by the change in the magnetizing force is measured in the same manner as before, 
and from this value the induction corresponding to the lower magnetizing force is readily calculated.

Data with negative values of the magnetizing force are obtained by reversing the currents in addition to making the adjustments already described. The points on the hysteresis curve may be taken in any order.

In this method of obtaining hysteresis data the measured quantity is the change in induction when the magnetization is changed at one step from a maximum to any other given point on the hysteresis loop. This method is comparatively free from the irregularities due to the slow creep that occurs when a magnetizing force is applied slowly or changed by small steps. It is also free from irregularities due to variations in the size of step in the "stepby-step" method. For these reasons and the fact that the errors are not cumulative, it seems the better method for standard measurements.

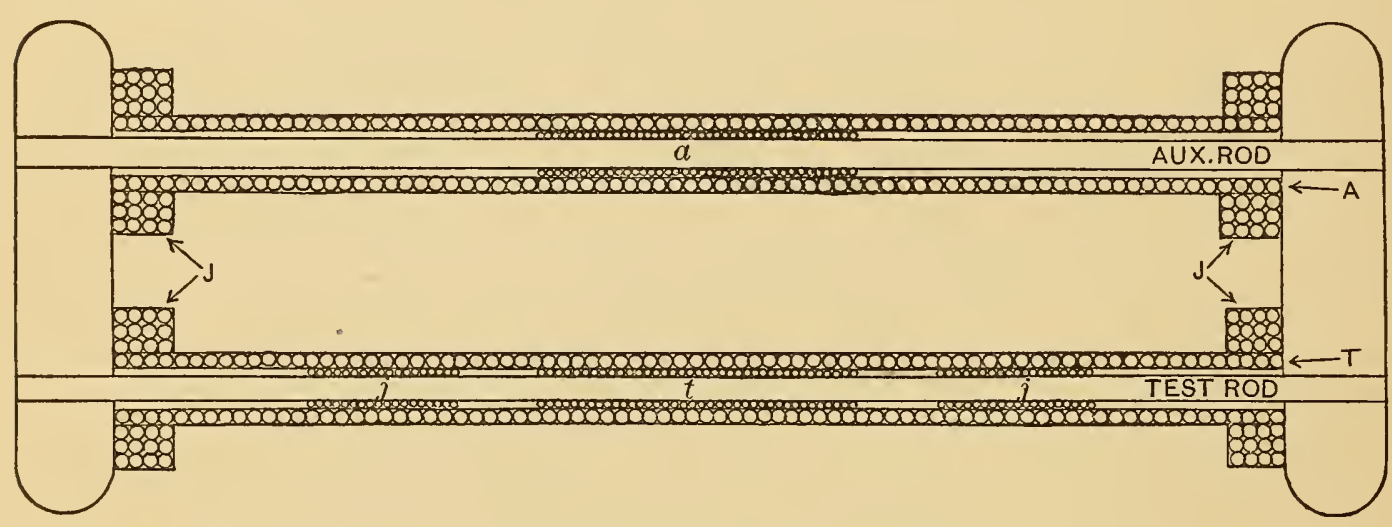

Fig. 28.- Showing the Relative Positions of Magnetizing and Test Coils.

$T$ and $A$ represent the main magnetizing solenoids.

$\mathrm{J}$, the compensating turns about the four joints.

$t$ and $a$ are the two test coils surrounding test and auxiliary specimen respectively.

$j$ is the end coil distributed with one-half over each end of the test rod.

Fig. 28 shows the relative positions of the magnetizing and test coils when double yokes and double rods are used. The lower rod is the one under test. The upper rod is an auxiliary rod of approximately the same magnetic properties as the test specimen. $T$ and $A$ are the two main magnetizing coils, one wound over each rod. Over the four joints are wound four short coils each about $\mathrm{I} .5 \mathrm{~cm}$ long. These are connected in series and used as a single coil. 

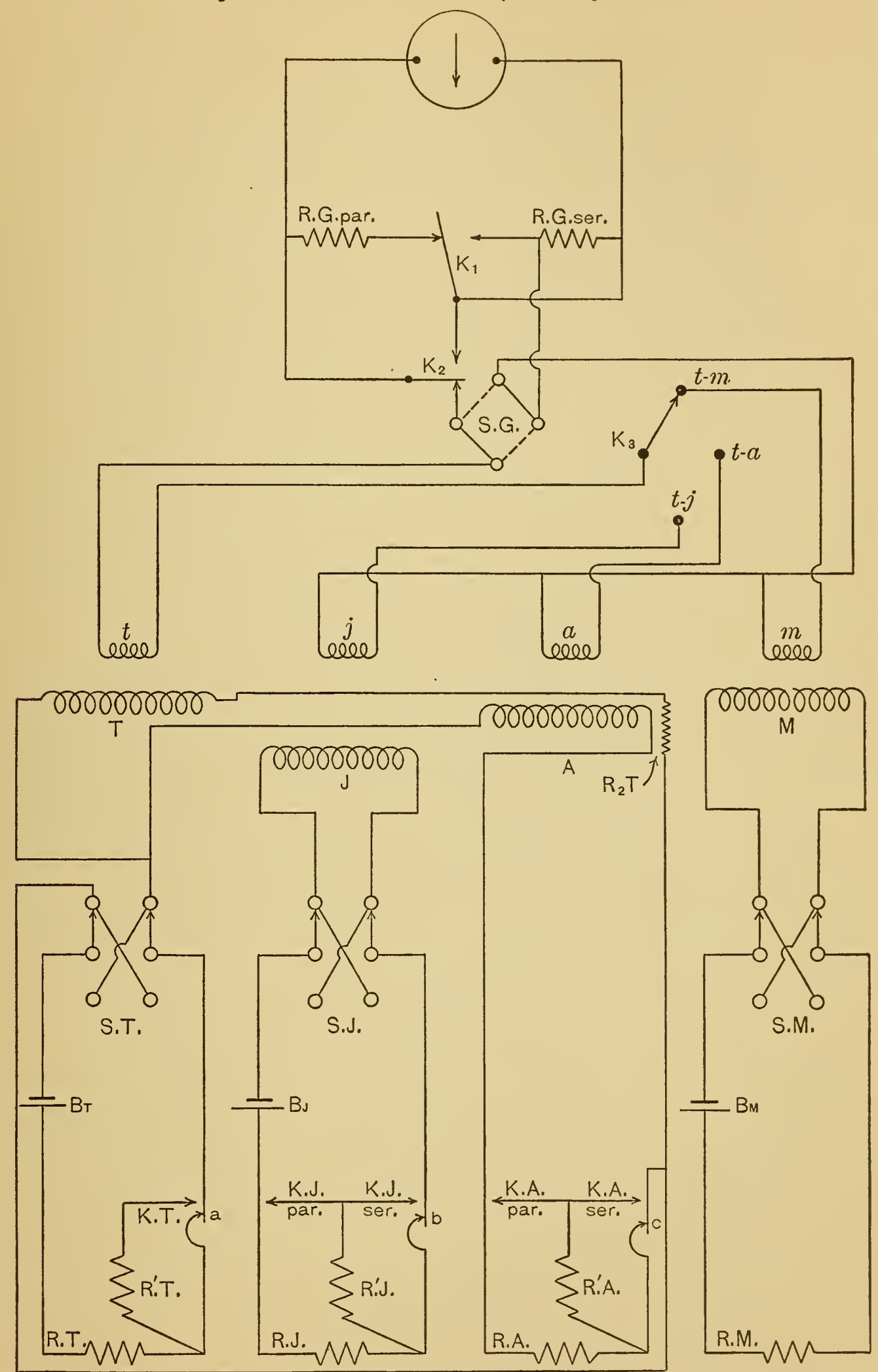

Fig. 29.-Showing Diagrammatically Fethull Battery and Galvanometer Connections used in Precision Magnetic Measurements.

$T$ refers to the main magnetizing circuit about the test bar, $\mathrm{J}$ to the compensating circuit, $\mathrm{A}$ to the magnetizing circuit about the auxiliary rod, and $M$ to the variable mutual induction circuit. 
The test coils $t$ and $a$ are wound over the middle portions of the test rod and auxiliary rod, respectively. Over the two ends of the test rod are wound the two halves of a third test coil $j$. These three test coils have the same number of turns and are spread over a considerable length of rod, so as to prevent any irregularities which may exist in the iron from exerting a preponderating influence. The end test coils are far enough away from the yokes and joints so that there is little irregularity from these causes. These test coils are wound on a separate form inside the solenoid, so that the correction for the flux between the rod and coil may be as small as possible.

Fig. 29 shows, diagrammatically, the full scheme of electric circuits both primary and secondary. The coils $T, J, A, t, j$, and $a$ are the same as those of the same letters in the preceding figure. $M$ and $m$ are the primary and secondary of the variable mutual inductance used to balance the emf. induced in the test coil.

The four reversing switches and the five keys in the various magnetizing circuits are all of the mercury type and are mounted on a single base and arranged side by side similar in size and position to the white keys of a piano, so that by using the fingers of both hands any combination may be operated. This compact arrangement of keys and switches should be borne in mind while reading the following description. On removing the fingers the keys are drawn back to their normal positions by means of small springs. The connections shown in the figure form a permanent set-up of considerable flexibility.

\section{FOR RINGS AND UNCOMPENSATED BARS AND YOKES.}

If this permanent set-up is to be used in magnetic measurements on rings or on uncompensated bar and yoke magnetic circuits, the magnetizing system consists solely of the circuit containing the battery $B_{\mathrm{T}}$. The circuit of battery $B_{\mathrm{J}}$ is not used. The induced electromotive force of the test coil $t$ acts on the galvanometer and produces a deflection when the switch $S T$ is reversed. If we wish to use the zero method, the switch $S M$ is reversed simultaneously and the resistances adjusted until the deflection on reversal is reduced to zero. 
In the galvanometer circuit the resistance $R G$ ser. determines the sensibility, and the resistance $R G$ par. adjusts to critical damping. The key $K_{1}$ is in the ordinary working position. If it is desired to use the galvanometer at its maximum sensibility, the key $K_{1}$ is thrown to the right, thus removing the shunt $R G$ par. and short circuiting the series resistance $R G$ ser.

The key $K_{2}$ is in its working position on the lower point, as shown. When raised to the upper contact, the test coils are thrown out of circuit and the galvanometer short-circuited. The reversing switch in the galvanometer circuit is very convenient in many cases.

If we are determining normal induction data in the above case the reversing switch $S T$ is the only one to be manipulated. To secure hysteresis data the change in induction when the flux in the iron passes from a maximum to some other value, positive or negative, is measured.

To lower the current from the maximum value to some other value the resistance $R^{\prime} T$ is inserted in series with the main resistance $R T$ by means of the key $K T$. The action of this key is to insert the resistance $R^{\prime} T$ across the contact $a$ and then open this contact. To change the current to a lower value of opposite sign the above operation is performed simultaneously with the reversal of switch $S T$.

\section{COMPENSATED BAR AND YOKE.}

In the determination of normal induction data in the bar and yoke magnetic circuit with compensation for yoke effects and dissimilarity in the test and auxiliary rods, all the circuits shown in the figure are used. The switches $S T$ and $S J$ are reversed repeatedly, and the resistance $R A$ and $R J$ adjusted until the three test coils, $t, j$, and $a$, indicate the same change in flux on reversal of the magnetizing currents. With the key $K_{3}$ on the point $t-a$, the equality of flux in the test and auxiliary rods is secured first. Then with the key $K_{3}$ on the point $t-j$ the flux near the magnetic joints is adjusted to uniformity. To measure the induction, $K_{3}$ is moved to the point $t-m$. A reversal of the magnetizing forces produces an impulsive electromotive force acting on the galvanometer, which may be measured as a deflection or may be compensated for as before by reversing simultaneously a 
suitable current through the variable inductance $M$. These operations are not so complicated as they appear at first sight. They have been much simplified by means of a set of special rocking mercury switches held in their normal position by suitable springs. These switches resemble in size, position, and operation the keys of a piano, and are operated as readily. Five of these keys can be operated with one hand with little more effort than is required for one. What complications exist are in the permanent apparatus. The operations themselves may be performed in less time than it takes to describe them.

To secure hysteresis data in the compensated bar and yoke method it is necessary to change the currents flowing in the coils $T$, $J$, and $A$ from the values necessary for adjustment for uniformity at the maximum induction to corresponding values at other inductions. The resistances and keys of the $T$ circuit are manipulated as in the case of a ring specimen. In circuit $J$, however, the second current may be greater or less than the first. For this reason it is necessary to be able to insert the resistance $R^{\prime} J$ either in series or in parallel with the initial resistance $R J$. By depressing the key $K J$ ser. first the resistance $R^{\prime} J$ is shunted around the contact $b$ and then the contact $b$ is broken. By depressing the key $K J$ par. one throws the resistance $R^{\prime} J$ in parallel with $R J$. In a similar manner the resistance $R^{\prime} A$ may be thrown either in series or in parallel with the resistance $R A$. Thus the resistances $R T$, $R J$, and $R A$ determine the magnitude and distribution of mmf. for the tip of a hysteresis loop, while $R^{\prime} T, R^{\prime} I$, and $R^{\prime} A$ determine the corresponding quantities for any other point of the cycle. By this means it is possible to adjust to uniformity of flux in any case that may arise. The variable mutual inductance may be used to reduce the measurement to a zero method or the direct deflections may be used.

Having secured uniformity of flux throughout the magnetic circuit we may measure the magnetizing current about each rod. If, further, the auxiliary rod is a standard of known properties, we have at the same time a comparison method as well as a direct measurement. Thus the one set of operations furnishes a check on itself, and no duplicate observations are necessary. 
The following is a sample set of data:

Formulæ use-

$$
\begin{aligned}
M & =\text { o.OI } A \\
B & =\operatorname{IOOOO} E_{\mathrm{M}} \\
H & =\operatorname{IOO} E
\end{aligned}
$$

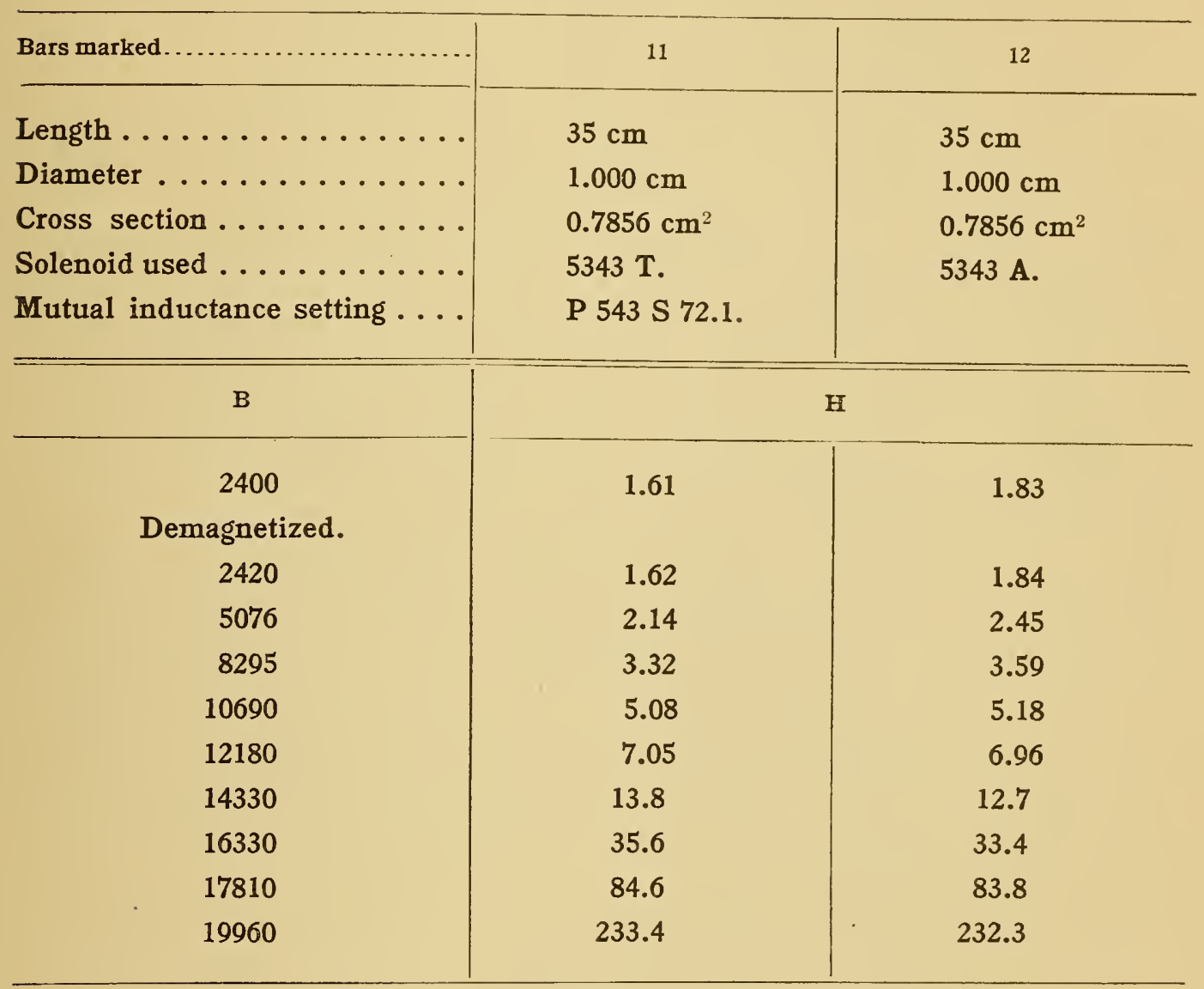

\section{GENERAL CONCLUSIONS.}

To sum up the general conclusions of the preceding investigations:

A close approximation to uniformity of flux along the test rod may be secured by using properly constructed specimens and yokes and properly distributed magnetizing coils.

The double bar and yoke form of magnetic circuit seems to offer the greatest number of advantages and the fewest disadvantages. The reluctance of the yoke and joints should be small. 
This is accomplished by having the yokes short and of moderately large cross section, and making the surface of contact between specimens and yoke of considerable area. The corners of the yokes should be rounded off so as to avoid, as far as possible, disturbing end effects.

The magnetomotive force may advantageously be distributed in three sections, a uniform solenoid over the test specimen, a second one over the auxiliary rod, and the third section subdivided into four short coils and placed over the four ends of the rods as near to the yokes as feasible. These three magnetomotive forces should be capable of independent adjustment and simultaneous reversal. The test coils to be used as indicators should be long enough to avoid errors due to local irregularities due to inhomogeneity of the rods. One coil should be wound under the middle section of each magnetizing solenoid and the third should be distributed with one-half over each end of the test bar, but not too near to the yokes.

In the measurement of the magnetizing force and the magnetic induction these quantities are obtained by zero methods directly from potentiometer readings.

As to accuracy, it was hoped at the beginning of this investigation that the magnetizing force required to produce any induction between $\mathrm{I}, 000$ and 20,000 gausses might be obtained to within I per cent. Investigations now in progress indicate that this accuracy has been secured.

Washington, May I, I909. 